\title{
Focal adhesion proteins Pinch1 and Pinch2 regulate bone homeostasis in mice
}

\author{
Yishu Wang, 1,2,3 Qinnan Yan, ${ }^{2,3}$ Yiran Zhao, ${ }^{2,3}$ Xin Liu, ${ }^{2,3}$ Simin Lin, ${ }^{2,3}$ Peijun Zhang, ${ }^{2,3}$ Liting Ma, ${ }^{2,3}$ \\ Yumei Lai, ${ }^{4}$ Xiaochun Bai, ${ }^{5}$ Chuanju Liu, ${ }^{6,7}$ Chuanyue Wu, ${ }^{8}$ Jian Q. Feng, ${ }^{9}$ Di Chen, ${ }^{4}$ Huiling Cao, ${ }^{2,3}$ \\ and Guozhi Xiao 2,3,4 \\ 1School of Life Science and Technology, Harbin Institute of Technology, Harbin, China. ${ }^{2}$ Guangdong Provincial Key \\ Laboratory of Cell Microenvironment and Disease Research, Shenzhen Key Laboratory of Cell Microenvironment, and \\ ${ }^{3}$ Department of Biology, Southern University of Science and Technology (SUSTech), Shenzhen, China. ${ }^{4}$ Department of \\ Orthopedic Surgery, Rush University Medical Center, Chicago, Illinois, USA. ${ }^{5}$ Department of Cell Biology, School of Basic \\ Medical Sciences, Southern Medical University, Guangzhou, China. ${ }^{6}$ Department of Orthopedic Surgery and ${ }^{7}$ Department \\ of Cell Biology, New York University School of Medicine, New York, New York, USA. ${ }^{8}$ Department of Pathology, University \\ of Pittsburgh, Pittsburgh, Pennsylvania, USA. ${ }^{9}$ Department of Biomedical Sciences, Texas A\&M University College of \\ Dentistry, Dallas, Texas, USA.
}

\begin{abstract}
Mammalian focal adhesion proteins Pinch1 and Pinch2 regulate integrin activation and cellextracellular matrix adhesion and migration. Here, we show that deleting Pinch1 in osteocytes and mature osteoblasts using the 10-kb mouse Dmp1-Cre and Pinch2 globally (double KO; dKO) results in severe osteopenia throughout life, while ablating either gene does not cause bone loss, suggesting a functional redundancy of both factors in bone. Pinch deletion in osteocytes and mature osteoblasts generates signals that inhibit osteoblast and bone formation. Pinch-deficient osteocytes and conditioned media from dKO bone slice cultures contain abundant sclerostin protein and potently suppress osteoblast differentiation in primary BM stromal cells (BMSC) and calvarial cultures. Pinch deletion increases adiposity in the BM cavity. Primary dKO BMSC cultures display decreased osteoblastic but enhanced adipogenic, differentiation capacity. Pinch loss decreases expression of integrin $\beta 3$, integrin-linked kinase (ILK), and $\alpha$-parvin and increases that of active caspase- 3 and -8 in osteocytes. Pinch loss increases osteocyte apoptosis in vitro and in bone. Pinch loss upregulates expression of both Rankl and Opg in the cortical bone and does not increase osteoclast formation and bone resorption. Finally, Pinch ablation exacerbates hindlimb unloadinginduced bone loss and impairs active ulna loading-stimulated bone formation. Thus, we establish a critical role of Pinch in control of bone homeostasis.
\end{abstract}

Conflict of interest: The authors have declared that no conflict of interest exists.

Copyright: (c) 2019, American Society for Clinical Investigation.

Submitted: July 11, 2019

Accepted: October 4, 2019

Published: November 14, 2019.

Reference information: JCI Insight.

2019;4(22):e131692.

https://doi.org/10.1172/jci.

insight.131692.

\section{Introduction}

In vertebrates, adult skeleton is a dynamic tissue that is constantly performing bone remodeling, a process in which the osteoclast-mediated bone resorption is closely coupled with the osteoblast-mediated bone formation. Bone remodeling allows skeleton to adapt its structure to mechanical loading by changing its size, shape, and strength; repairing damaged bone; and maintaining a proper bone mass to support bone homeostasis. Abnormal bone remodeling causes metabolic bone diseases, such as osteoporosis and osteopetrosis. Elucidating mechanisms that regulate bone remodeling is likely to reveal new approaches to the prevention and treatment of these diseases.

Osteocytes originate from osteoprogenitors through osteoblast differentiation and are the most abundant bone cell, comprising $90 \%-95 \%$ of total bone cells. Osteocytes play an important role in regulating bone remodeling under normal physiological and diseased states (1-7). Osteocytes communicate with each other and with osteoclasts and osteoblasts on the bone surfaces through their dendrites. Osteocytes produce several important soluble factors that regulate osteoclast and osteoblast formation and function. Among those are sclerostin, the activator of NF-kB ligand (RANKL), and osteoprotegerin (OPG) (8-11). Osteocytes are the main source of the fibroblast growth factor 23 (FGF23), a major regulator of phosphate homeostasis (12). Mechanical forces are the most potent anabolic stimulus of the skeleton. Mechanical loading from daily movement, exercise, and gravity increases bone mass and strength and improves bone 
architecture, while skeletal unloading leads to dramatic bone loss. Osteocytes are believed to be a main mechano-sensor of the bone. Osteocytes reside in the lacunae throughout the mineralized bone matrix and are well positioned to sense mechanical signals and transduce them to biochemical signals, which are eventually integrated into cellular responses that regulate bone remodeling $(2-6,13,14)$. Osteocytes die through apoptosis (15). However, key signals that control osteocyte function and survival are poorly defined.

Mammalian cells have 2 functional PINCH proteins, PINCH1 (LIMS1) and PINCH2 (LIMS2). They are 5 LIM domain-containing proteins that play important roles in cytoskeletal organization and cell-extracellular matrix adhesion, migration, proliferation, and survival (16-21). Both PINCH1/2 are ubiquitously expressed in most mammalian tissues and organs, including the heart, lung, liver, kidney, and bladder (20). Global deletion of Pinch1 in mice causes lethality (17), while Pinch2-KO mice have no apparent phenotypes $(19,22)$. Pinch proteins exert their functions in part by forming several distinct functional protein-protein complexes, including the ILK-Pinch-parvin (IPP), Pinch-Nck2, and Pinch-Rsu1 complexes (18, 20, 23-26). For example, the IPP complex regulates cell contractility and cytoskeletal dynamics in mice (21, 27-29). Previous studies were primarily focused on roles of Pinch in regulation of multiple properties of cancers, such as growth, survival, apoptosis, progression, invasion, and radio- and chemo-resistance (19, 20, 30-33).

Important roles of focal adhesion proteins in organogenesis have begun to emerge. We demonstrate that the focal adhesion protein Kindlin-2 — which interacts with integrin-linked kinase (ILK), part of the IPP complex - regulates chondrogenesis and early skeletal development by modulating TGF- $\beta$ signaling and Sox9 expression in chondrocytes and their precursors (34). We further demonstrate that Kindlin-2 directly promotes osteoblastic, but inhibits adipogenic, differentiation of mesenchymal stem cells through control of Yap1/Taz (35). Liang and coworkers reported that Pinch proteins are critical for cardiomyocyte function and heart development in mice. Deletion of both Pinch1/2 in the heart resulted in severe dilated cardiomyopathy and death due to heart failure in mice (36). However, roles of Pinch1/2 in bone have not been defined.

In this study, we deleted Pinch1 expression using the mouse 10-kb Dmp1-Cre transgene, which primarily targets osteocytes and mature osteoblasts, with and without global Pinch2 deletion in mice. Through comprehensive analyses of cells and tissues of the double and single mutant mice, we establish a critical role of Pinch $1 / 2$ and a functional redundancy of both factors in control of bone homeostasis through distinct mechanisms.

\section{Results}

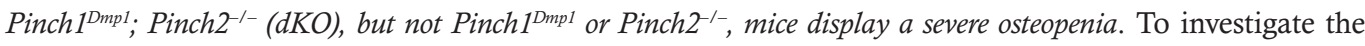
roles of Pinch1/2 in bone, we generated mice lacking Pinch1 expression in mature osteoblasts and osteocytes by breeding the floxed Pinch1 mice (Pinch $1^{f / f f}$ ) with the $10-\mathrm{kb}$ mouse dentin matrix protein $1-\mathrm{Cre}$

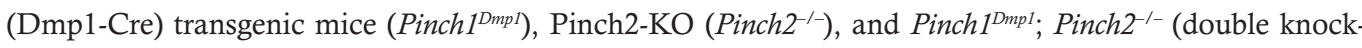
out; dKO) (Figure 1, A-D). Results from Western blotting, quantitative PCR (qPCR), and IHC staining analyses demonstrated that Pinch1 expression was significantly reduced in bone and in osteocytes embedded in the cortical bone matrix of $\mathrm{dKO}$ mice compared with control mice (Dmp1-Cre). To determine the effects of Pinch deletion on bone mass, we performed micro-computed tomography $(\mu \mathrm{CT})$ analysis of distal femurs of 3-month-old female control (Dmp1-Cre), Pinch1 ${ }^{\text {Dmp1 }}$, Pinch2 ${ }^{--}$, and dKO mice. The bone mineral density (BMD) and bone volume/tissue volume (BV/TV) fraction (BV/TV) were not significantly different among the first 3 groups. However, both parameters were dramatically decreased in dKO mice compared with age- and sex-matched control (Dmp1-Cre) or single mutant mice (Pinch1 $1^{\text {Dmpl }}$ or Pinch2-/-) (Figure 1, A-C). The cortical thickness (Ct.Th) was not altered by the double or single mutations (Figure 1D). Specifically, the values of BMD and BV/TV were decreased by $52 \%$ and $60 \%$, respectively, in $\mathrm{dKO}$ mice compared with those of control mice. Consistent with results from $\mu \mathrm{CT}$ analyses, results from $\mathrm{H} \& \mathrm{E}$ staining of tibial sections of 6-month-old female mice showed much less trabecular bone in $\mathrm{dKO}$ mice than in controls (Figure 1E). We performed qPCR analysis using RNA from the osteocyte-enriched femoral drafts (with their BM flushed) from 6-month-old female control and $\mathrm{dKO}$ mice and observed that the level of Pinch1 mRNA was dramatically reduced in dKO relative to control femurs (Figure 1F). Similarly, results from Western blotting using protein extracts from femoral drafts revealed that the level of Pinch1 protein was decreased in $\mathrm{dKO}$ relative to control bones (Figure 1G). These reductions are specific because expression of Pinch1 in nonbone tissues, such as heart, lung, spleen and kidney, was not reduced in dKO versus control mice (Figure 1, F and G). Results from IHC staining of the longitudinal tibial sections of 6-month-old female control and $\mathrm{dKO}$ mice revealed fewer Pinch1-expressing osteocytes embedded in the 
A
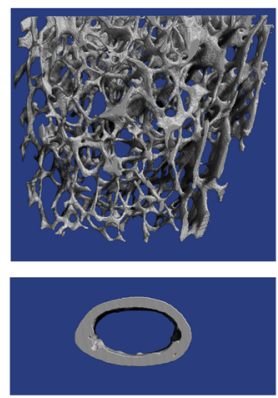

Dmp1-Cre
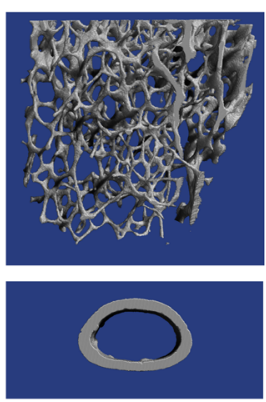

Pinch2-1-
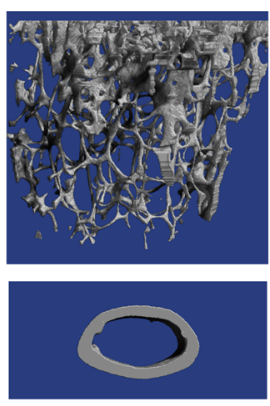

Pinch1 ${ }^{\text {Dmp1 }}$

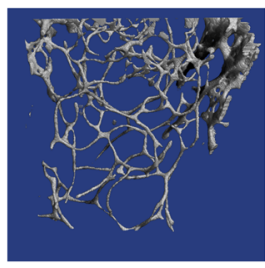

B

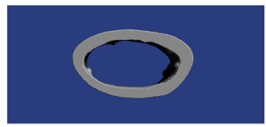

Pinch1 $^{\text {Dmp1 }} ;$ Pinch̄ $^{-1-}$
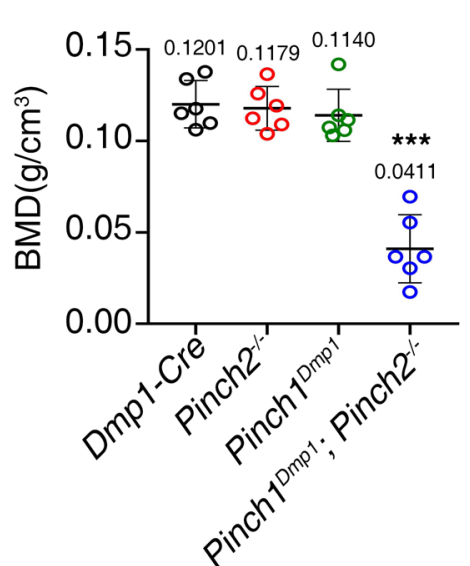

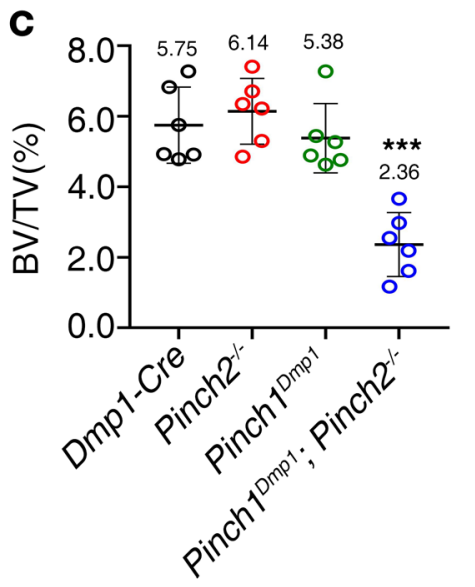

$\mathbf{F}$

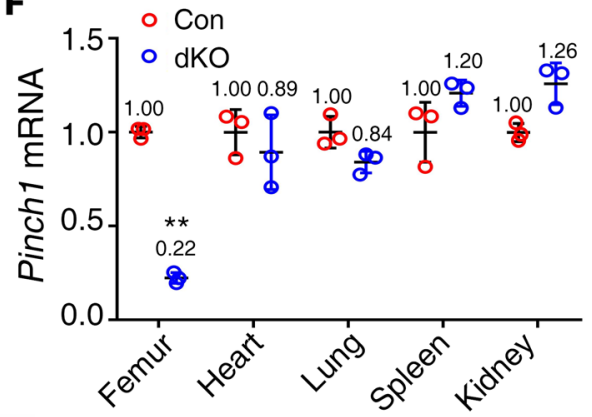

H

IHC: Pinch1

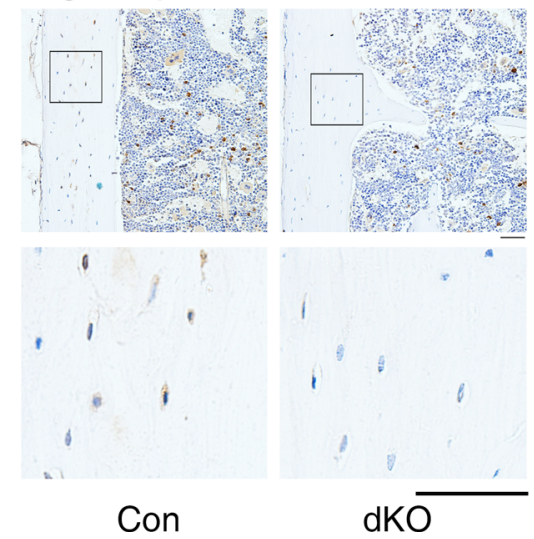

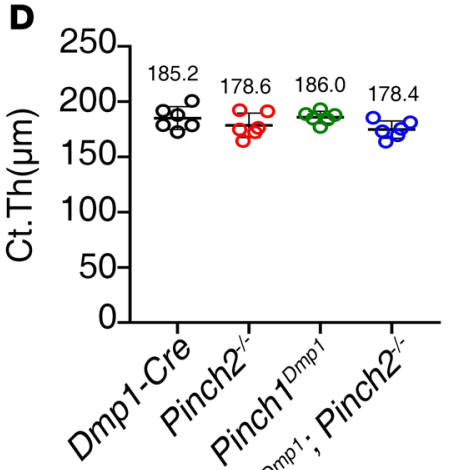

E

$H \& E$

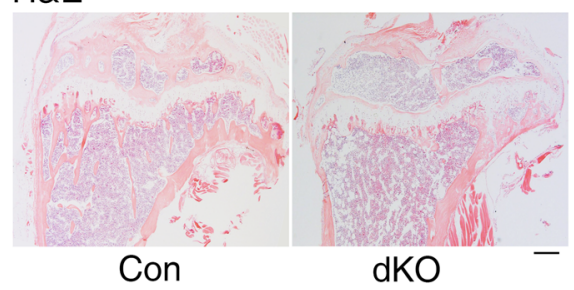

G

$\frac{\text { Femur }}{12} \frac{\text { Heart }}{12} \frac{\text { Liver }}{12} \frac{\text { Lung }}{12} \frac{\text { Spleen }}{12} \frac{\text { Kidney }}{12}$

Pinch1

Gapdh

1.Con; 2.dKO

I

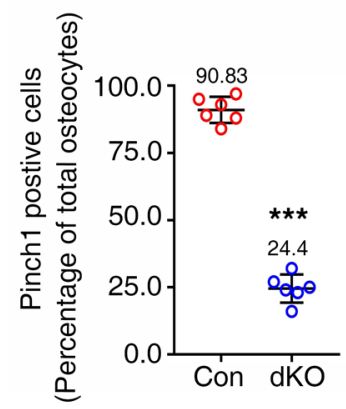

$\mathbf{J}$

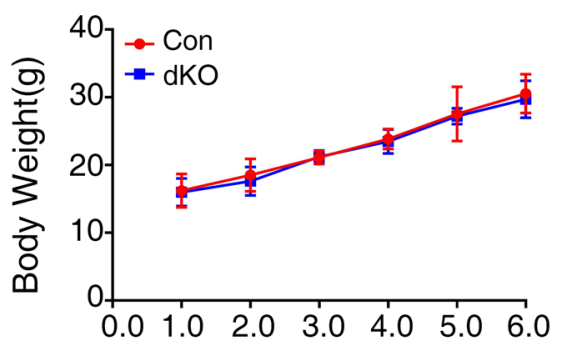


Figure 1. Pinch1 ${ }^{\text {Dmp1; }}$ Pinch2 ${ }^{-/-}$(dKO), but not Pinch1 ${ }^{\text {mp1 }}$ or Pinch2-/-, mice display a low bone mass. (A) Three-dimensional (3-D) reconstruction from micro-computerized tomography $(\mu \mathrm{CT})$ scans of distal femurs from 3-month-old female control (Dmp1-Cre), Pinch1 $1^{\mathrm{mp1}}$, Pinch $2^{-1-}$, and dKO mice. Scale bar: $100 \mu \mathrm{m}$. (B-D) Quantitative analyses of bone mineral density (BMD) (B), bone volume/tissue volume (BV/TV) (C), and cortical thickness (Ct.Th) (D) of distal femurs from $\mathbf{A}$. $n=6$ mice per group. ${ }^{* *} P<0.001$ vs. controls, 2-way ANOVA. Results are expressed as mean \pm SD. (E) H\&E staining of tibial sections of 6-month-old female control and dKO mice. (F) qPCR analyses. Total RNAs isolated from 3-month-old female middiaphyseal femoral shafts (with their BM flushed) and other indicated tissues were used for qPCR analysis for expression of Pinch1 gene, which was normalized to Gapdh mRNA. $n=3$ mice per group. ${ }^{* *} P<0.01$ vs. controls, unpaired Student's $t$ test. Results are expressed as mean \pm SD. (C) Western blot analysis. Protein extracts were isolated from osteocyte-enriched middiaphyseal femoral shafts (with their BM flushed) and other indicated tissues of 6-month-old female mice of control and dKO mice and subjected to Western blotting using an antibody against Pinch1. Western blotting was repeated 3 times. (H) IHC staining. Longitudinal tibial sections of 6-month-old female control and dKO mice were stained with an antibody against Pinch1. (I) Quantification of H. $n=6$ mice per group. ${ }^{* *} P<0.001$ vs. control. Unpaired Student's t test. Results are expressed as mean \pm SD. (J) Growth curve. $n=6$ mice per group. Results are expressed as mean \pm SD.

bone matrix in $\mathrm{dKO}$ bone than in control bones (Figure 1, H and I). The Dmp1-Cre (control), Pinch1 ${ }^{\text {Dmpl }}$, Pinch $2^{--}$, and $\mathrm{dKO}$ mice were viable, fertile, and born with the expected Mendelian frequency. The body weights were not different between control and $\mathrm{dKO}$ mice (Figure $1 \mathrm{~J}$ ).

Pinch deletion causes severe osteopenia in 6- and 14-month-old mice. We next investigated the effects of Pinch

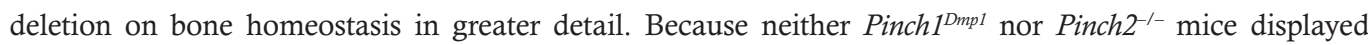
marked skeletal defects, we next focused our study on analyzing the phenotypes of $\mathrm{dKO}$ mice in comparison with Dmp1-Cre mice (controls). We harvested femurs from mice of the 2 genotypes of different ages and sexes. $\mu \mathrm{CT}$ analysis of distal femurs of those mice revealed dramatic decreases in BMD and BV/TV in $\mathrm{dKO}$ mice compared with control mice at different ages and sexes (Figure 2A). Specifically, BMD was decreased by $47 \%$ in 6 -month-old and $45 \%$ in 14 -month-old female $\mathrm{dKO}$ mice (Figure $2 \mathrm{~B}$ ), and BV/TV was reduced by $60 \%$ at both ages in $\mathrm{dKO}$ mice compared with those in sex-matched control littermates (Figure $2 \mathrm{C}$ ). The trabecular number (Tb.N) was significantly decreased, while the trabecular separation (Tb.Sp) was increased in $\mathrm{dKO}$ mice relative to control littermates (Figure 2, D and E). In contrast, the trabecular thickness (Tb. $\mathrm{Th}$ ) and Ct.Th were not significantly reduced in $\mathrm{dKO}$ mice compared with those in control mice (Figure 2, D-G). A similar severe osteopenia was observed in 6-month-old male dKO mice (Figure 2, A-G).

Pinch loss impairs the mechanical properties of $d K O$ femurs. We next performed the 3-point bending tests on femurs of 4-month-old control and dKO female mice to determine whether Pinch loss impairs the mechanical properties of the cortical bones. The results revealed that the values for both stiffness and maximum load were significantly reduced in dKO versus control mice (Figure 2, $\mathrm{H}$ and I).

Taken together, these results demonstrate an important role of Pinch $1 / 2$ and a functional redundancy of the 2 factors in control of bone mass and function.

Reduced osteoblast formation and function contribute to the osteopenia in dKO mice. To investigate mechanisms through which Pinch loss causes osteopenia, we performed several sets of experiments. We measured the serum level of procollagen type 1 amino-terminal propeptide (P1NP), an in vivo indicator for osteoblast function and bone formation, and found that it was dramatically reduced in 6-month-old $\mathrm{dKO}$ mice compared with that of control mice (Figure 3A). We determined the in vivo bone-forming activity by performing a calcein double-labeling experiment and found that the mineralization apposition rate (MAR) and bone formation rate (BFR) in the tibial metaphyseal cancellous bones were significantly reduced in 3-month-old female dKO mice compared with those in control mice (Figure 3, B-D). To determine the effects of Pinch loss on the osteoid production and mineralization, we performed the von Kossa staining of tibial sections of 3-month-old female control and $\mathrm{dKO}$ mice and found that the osteoid volume/tissue volume (OV/TV) and mineralized bone volume/tissue volume (mBV/TV) were significantly decreased in $\mathrm{dKO}$ mice relative to those in control mice (Figure 3, E-G). Results from Western blotting analyses using tibial protein extracts revealed reduced expression of Osx protein, a marker for osteoblasts and their precursors in $\mathrm{dKO}$ relative to control bones (Figure $3, \mathrm{H}$ and $\mathrm{I}$ ).

Pinch deletion does not increase osteoclast formation and bone resorption. We further investigated whether Pinch loss impacts the osteoclast formation and bone resorption. We first performed the tartrate-resistant acid phosphatase (TRAP) staining of tibial sections for osteoclast formation in bone and found that the osteoclast number/bone perimeter (Oc.Nb/BPm), but not osteoclast surface/bone surface (Oc.S/BS), was increased in both primary and secondary spongiosa bones in $\mathrm{dKO}$ mice compared with those of controls; however, the increases did not achieve a statistical significance (Figure 4, A-E). Furthermore, the serum levels of the collagen type I cross-linked C-telopeptide (CTX1), degradation products from type I collagen during in vivo osteoclastic bone resorption, were not significantly altered in 6-month-old female $\mathrm{dKO}$ mice relative to control mice, as 
A

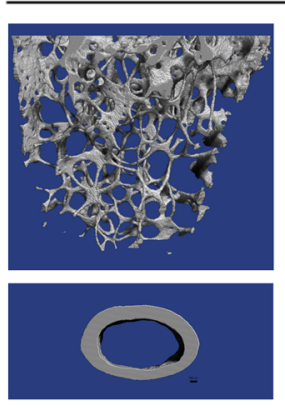

B

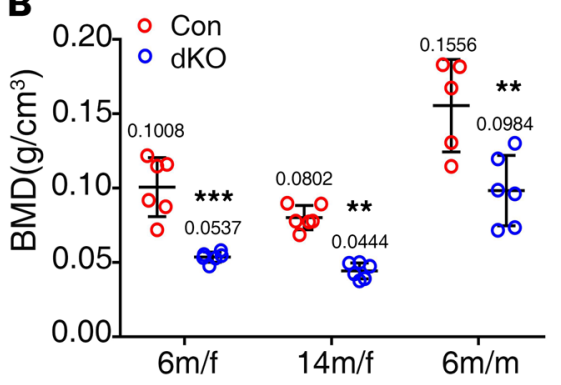

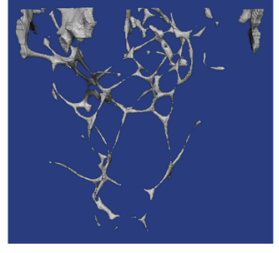

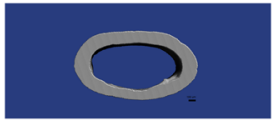

$14 \mathrm{~m} / \mathrm{female}$
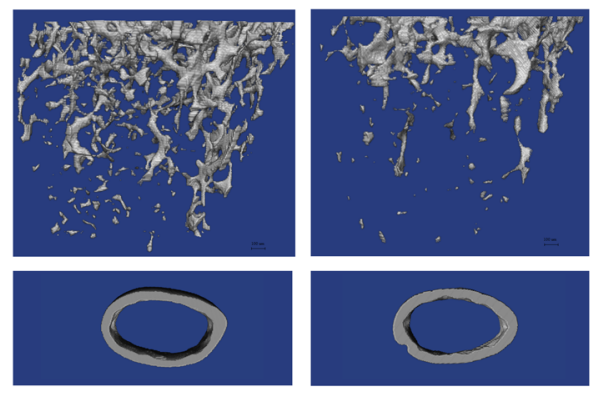

$6 \mathrm{~m} / \mathrm{male}$
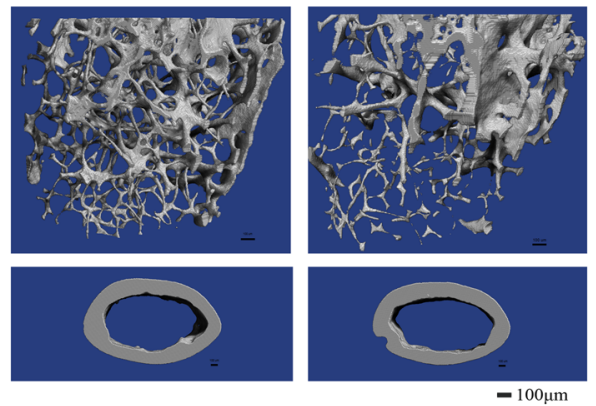

C

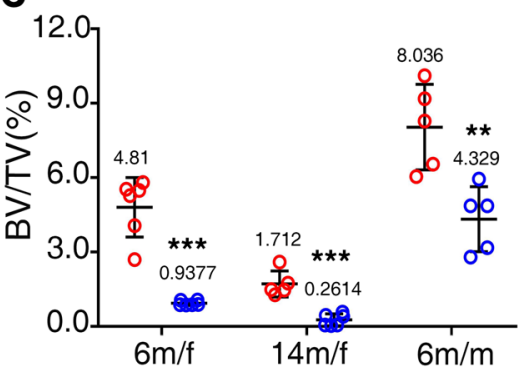

D

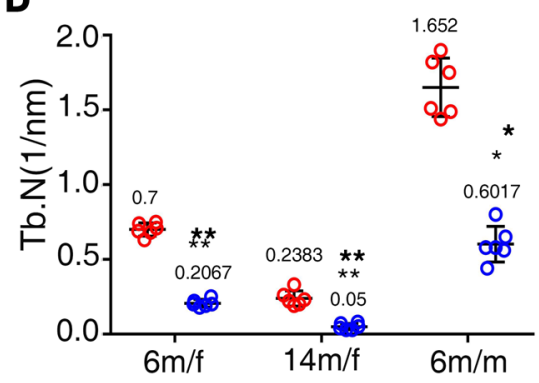

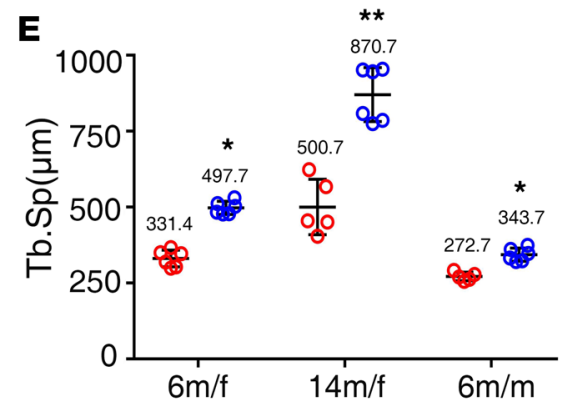

H

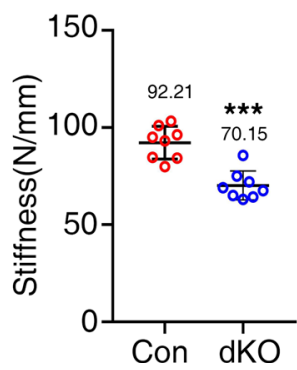

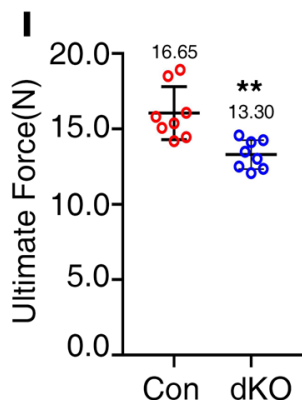

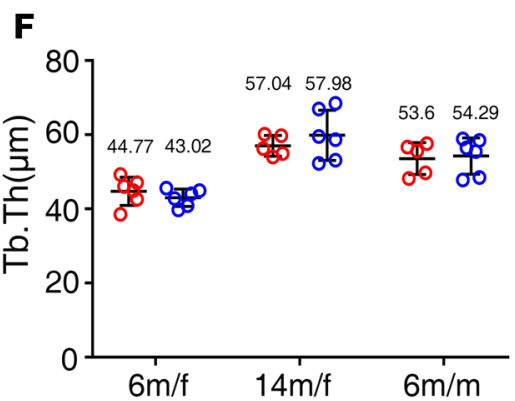

G

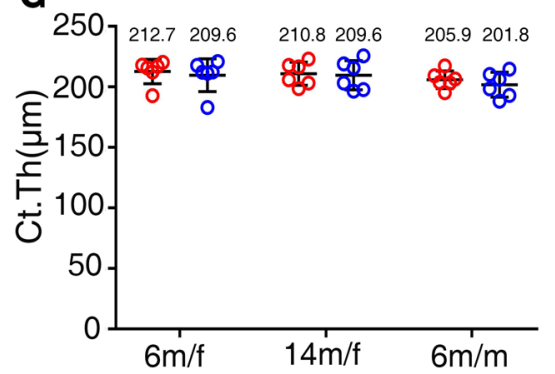

Figure 2. Pinch loss causes severe osteopenia in 6- and 14-month-old male and female mice. (A) Three-dimensional (3-D) reconstruction from micro-computerized tomography $(\mu C T)$ scans of distal femurs from 6- and 14-month-old female and 6-month-old male control and dKO mice. Scale bar: $100 \mu \mathrm{m}$. (B-C) Quantitative analyses of bone mineral density (BMD), bone volume/tissue volume (BV/TV), trabecular number (Tb.N), trabecular thickness (Tb.Th), trabecular separation (Tb.Sp), and cortical thickness (Ct.Th) of distal femurs from A. $n=6$ mice per group. ${ }^{*} P<0.05,{ }^{* *} P<0.01,{ }^{* * *} P<0.001$ vs. controls. Unpaired Student's $t$ test. Results are expressed as mean $\pm \mathrm{SD}$. (H and $\mathbf{I})$ Three-point bending tests. Femurs isolated from 4-monthold control and dKO female mice were subjected to the 3-point bending test as described in Materials. Stiffness (H) and maximum load (I). $n=8$ mice per group. ${ }^{* *} P<0.01,{ }^{* *} P<0.001$ vs. controls. Unpaired Student's $t$ test. Results are expressed as mean \pm SD.

measured by ELISA assay (Figure 4F). Results from Western blotting showed that the level of secreted Rank was increased in $\mathrm{dKO}$ cortical bones relative to that in control cortical bones (Figure 4G). Results from IHC staining revealed increased expression of Rankl protein in $\mathrm{dKO}$ osteocytes relative to control osteocytes in bones (Figure 4H). Results from reverse transcription PCR (RT-PCR) analyses showed that the levels of both Rankl and Opg mRNA were similarly upregulated in dKO versus control bones (Figure 4I).

Pinch deletion increases sclerostin expression in osteocytes embedded in the cortical bone matrix. Sclerostin, the Sost gene protein product almost exclusively expressed by osteocytes (8), binds to the Wnt coreceptors Lrp5 and Lrp6 and inhibits the Wnt/ $\beta$-catenin signaling, osteoblast function, bone formation $(37,38)$. Interestingly, results from Western blotting revealed that expression of sclerostin was increased in $\mathrm{dKO}$ bones compared with that of control bones (Figure 5, A and B). Results from IHC staining confirmed the upregulation of sclerostin in osteocytes embedded in $\mathrm{dKO}$ cortical bone matrix (Figure 5C). 
A

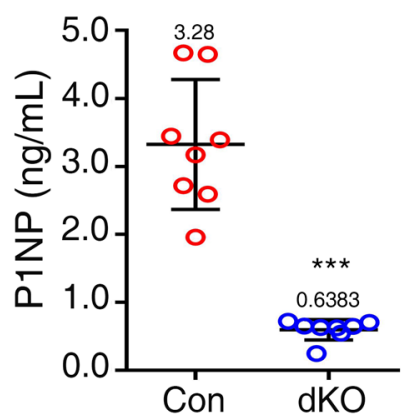

B

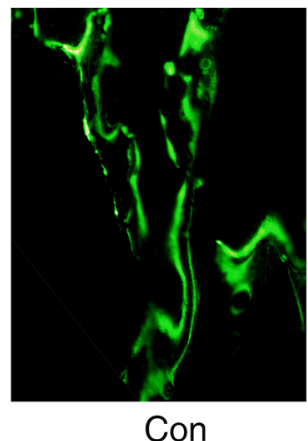

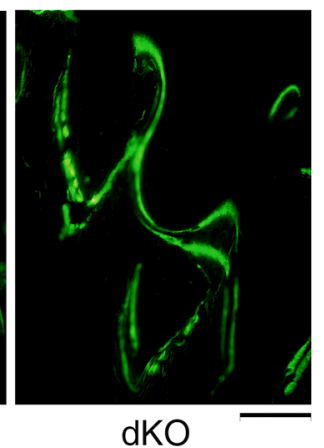

C

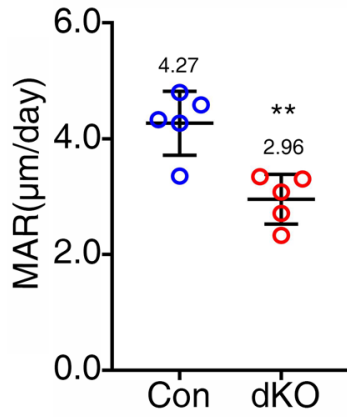

D

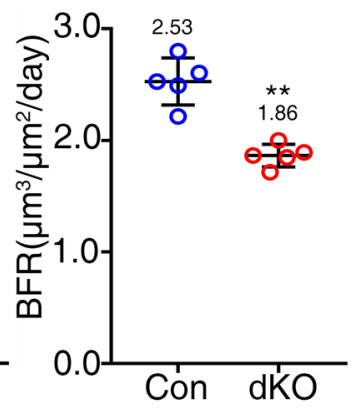

E

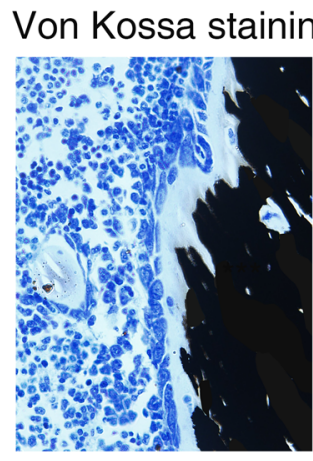

Con
$\mathbf{F}$

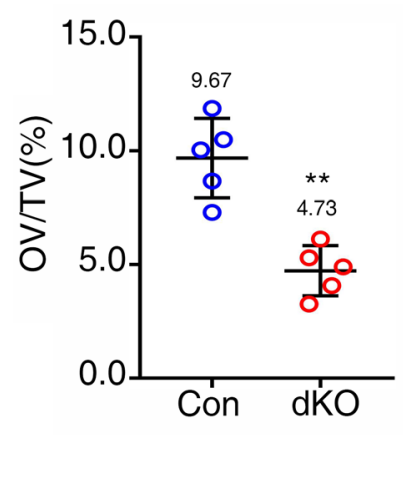

G

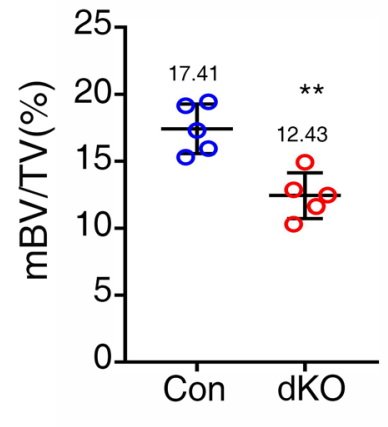

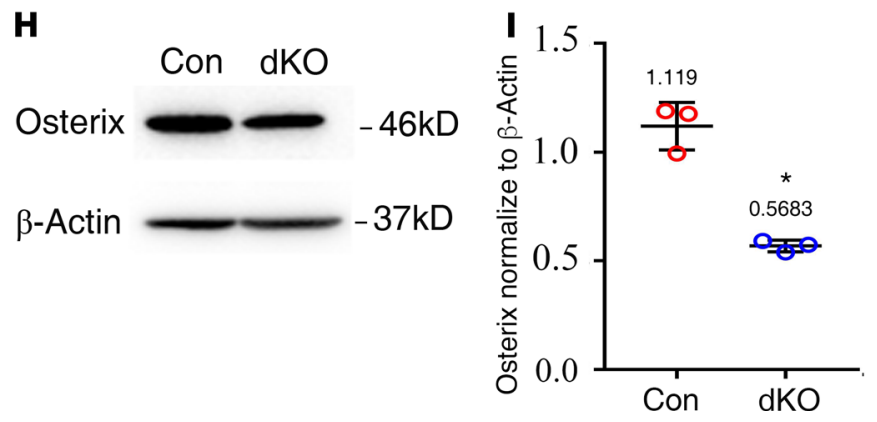

Figure 3. Pinch loss impairs osteoblast formation and function in bone. (A) Serum level of procollagen type 1 amino-terminal propeptide (P1NP). Sera were collected from 6 -month-old female mice of the 2 genotypes. $n=8$ mice per group. ${ }^{* * *} P<0.001$ vs. controls. Unpaired Student's $t$ test. Results are expressed as mean \pm SD. (B-D) Calcein double labeling. Representative images of 3-month-old female control and dKO tibial sections (B). Sections of nondemineralized tibiae of 3-month-old female control and dKO mice were used for measurements of mineralization apposition rate (MAR)of the metaphyseal trabecular bones (C) and bone formation rate (BFR) (D). $n=5$ mice per group. ${ }^{* *} P<0.01$ vs. controls. Unpaired Student's $t$ test. Results are expressed as mean \pm SD. Scale bar: $50 \mu \mathrm{m}$. (E-G) von Kossa staining. Undecalcified sections of femora from 3-month-old female control and dKO mice were subjected to von Kossa staining (E). Quantitative osteoid volume/tissue volume (OV/TV) (F) and mineralized bone volume/tissue volume (mBV/TV) (G) data for the cancellous bones from distal femora were measured by bone morphometry. $n=5$ mice per group. ${ }^{* *} P<0.01 \mathrm{vs}$. controls. Unpaired Student's $t$ test. Results are expressed as mean \pm SD. Scale bar: $50 \mu \mathrm{m}$. (H) Western blot analyses. Protein extracts were isolated from femurs of 6-month-old female control and dKO mice and subjected to Western blotting using an antibody against osterix. (I) Quantitative data. ${ }^{*} P<0.05$ vs. controls. Unpaired Student's $t$ test. Results are expressed as mean $\pm \mathrm{SD}$. Western blotting was repeated 3 times.

Conditioned media from $\mathrm{dKO}$ bone slice cultures contain abundant sclerostin protein and suppresses osteoblast differentiation in primary BMSC and calvarial osteoblast cultures. Although above results showed increased expression of sclerostin in osteocytes of $\mathrm{dKO}$ bones, we did not observe a significant increase in serum sclerostin in $\mathrm{dKO}$ mice compared with control mice (Figure 5D). We next prepared the conditioned media (CM) from ex vivo-cultured middiaphyseal femoral and tibial slices from 6-month-old control and $\mathrm{dKO}$ mice and observed a dramatic increase in sclerostin in $\mathrm{dKO} \mathrm{CM}$ compared with control CM (Figure 5E). Primary BM stromal cells (BMSCs) were isolated from normal C57BL/6 mice and treated with the CM under osteoblast differentiation condition for 7 days. The results showed that 
A
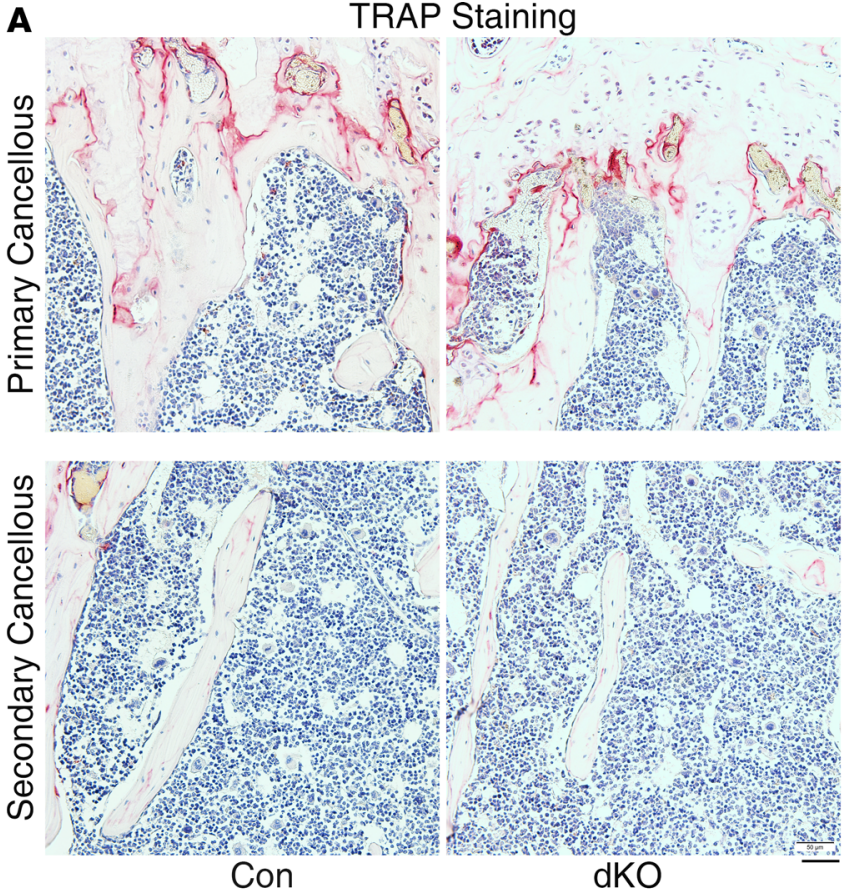

Con

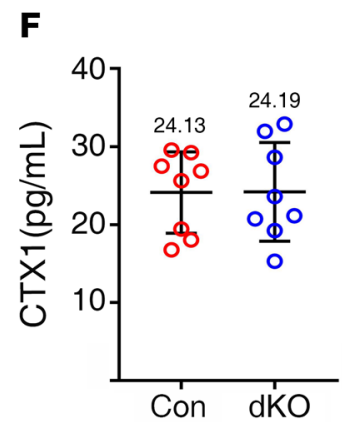

G

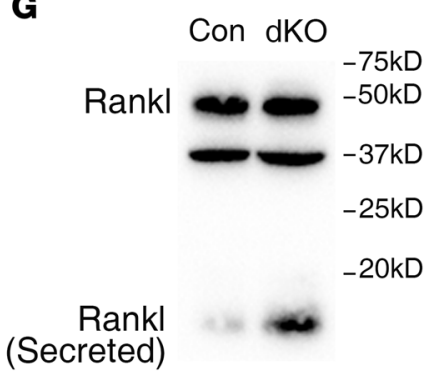

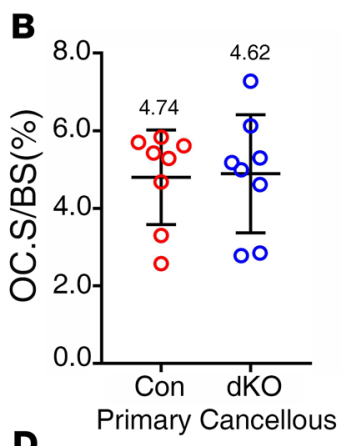

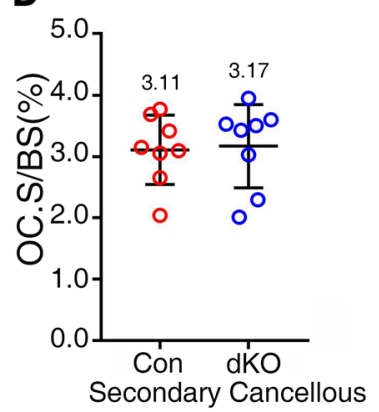

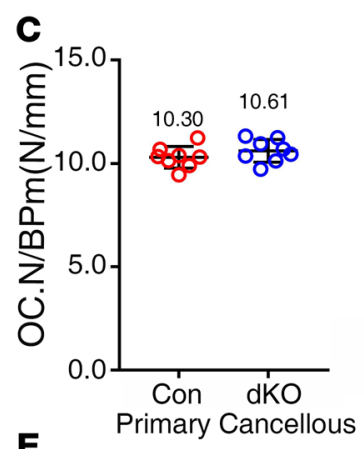

E

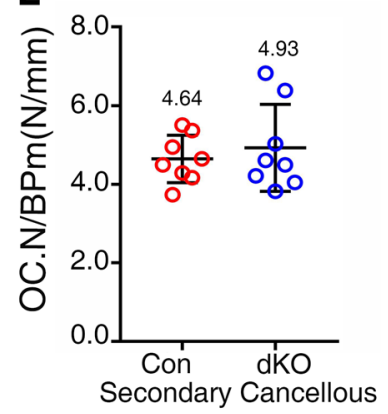

H IHC: Rankl
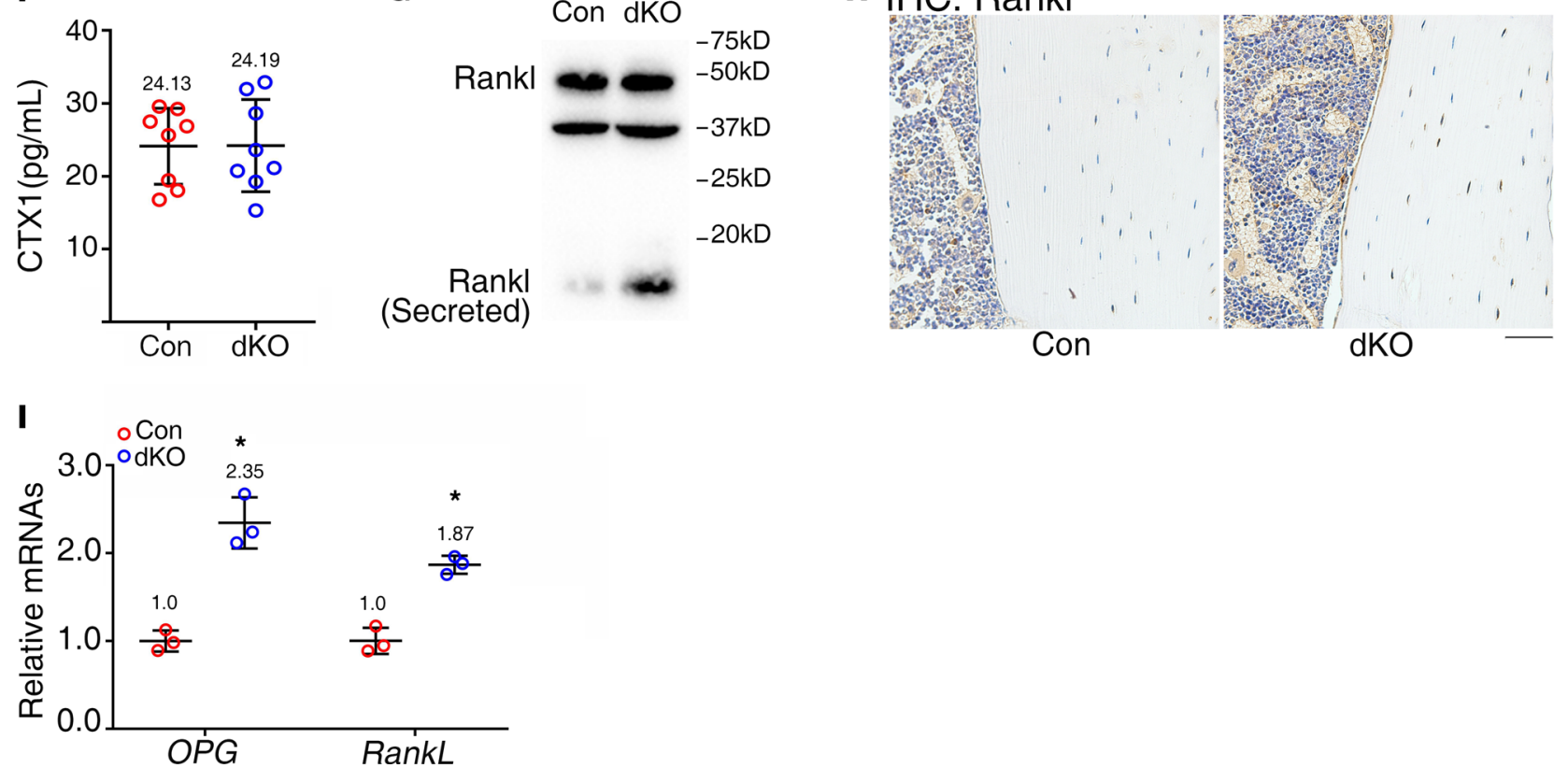

Figure 4. Pinch loss does not impair osteoclast formation and bone resorption. (A-E) Tartrate-resistant acid phosphatase (TRAP) staining. Tibial sections of 6-month-old female control and dKO mice were used for TRAP staining (A). Osteoclast surface/bone surface (Oc.S/BS) (B and D) and osteoclast number/bone perimeter (Oc.N/BPm) (C and $\mathbf{E}$ ) of primary and secondary spongiosa bones of the 2 genotypes were measured using ImagePro Plus 7.0. Scale bar: $50 \mu \mathrm{m} . n=8$ mice per group. Unpaired Student's $t$ test. Results are expressed as mean \pm SD. (F) Serum level of collagen type I cross-linked C-telopeptide (CTX1). Sera were collected from 6-month-old female control and dKO mice and subjected to ELISA assay for CTX1. $n=8$ mice per group. Unpaired Student's $t$ test. Results are expressed as mean \pm SD. (C) Western blot analysis. Protein extracts were isolated from osteocyte-enriched middiaphyseal femoral shafts (with their BM flushed) of 6-month-old female control and dKO mice and subjected to Western blotting using an anti-Rankl antibody. Western blotting was repeated 3 times. (H) IHC staining of tibial sections of 6-month-old female control and dKO mice with an anti-Rankl antibody. Scale bar: $50 \mu \mathrm{m}$. (I) qPCR analyses. Total RNAs were isolated from femurs of 6-month-old female control and dKO mice and used for qPCR analysis for expression of Rankl and Opg genes, which were normalized to Gapdh mRNA. $n=3$ mice per group. ${ }^{*} P<0.05$ vs. controls. Unpaired Student's $t$ test. Results are expressed as mean \pm SD. 
A

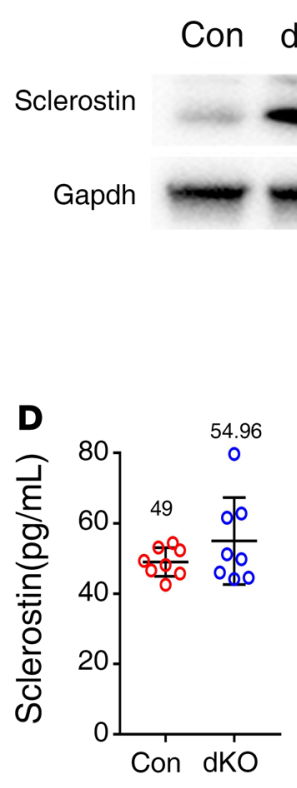

B

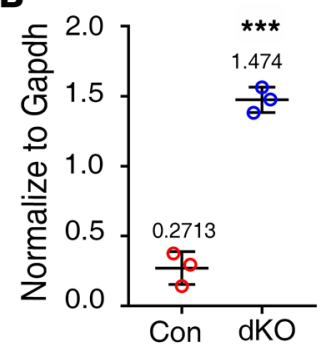

C IHC: Sclerostin

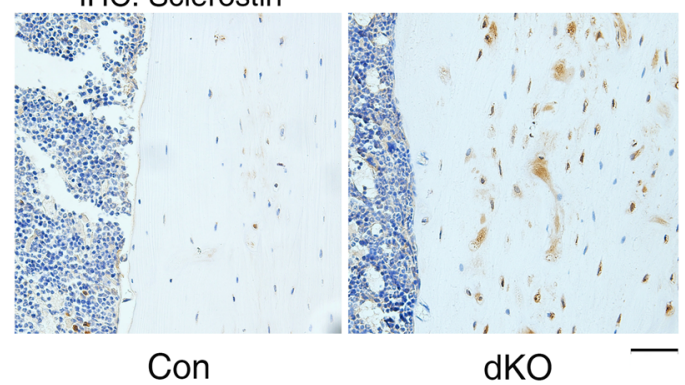

E

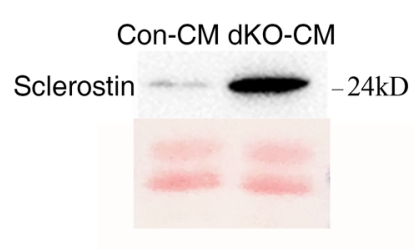

H

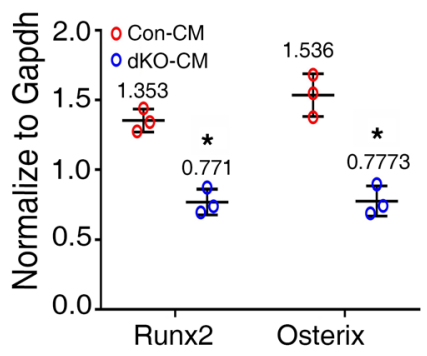

F

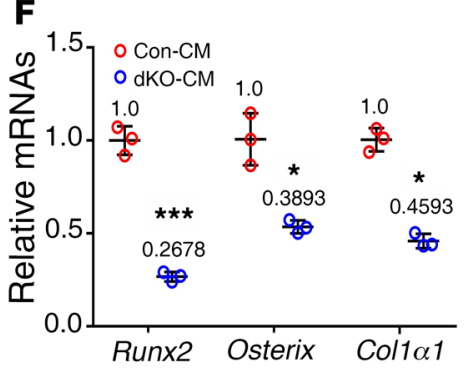

G

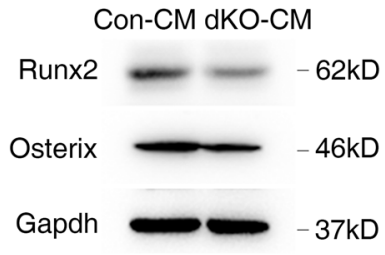

$\mathbf{K}$

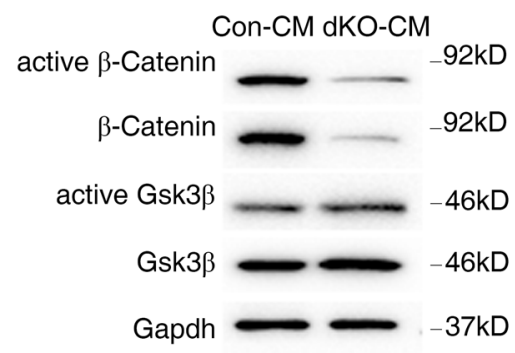

I

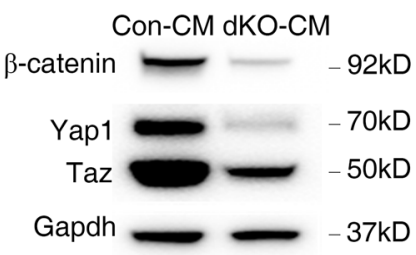

$\mathbf{J}$

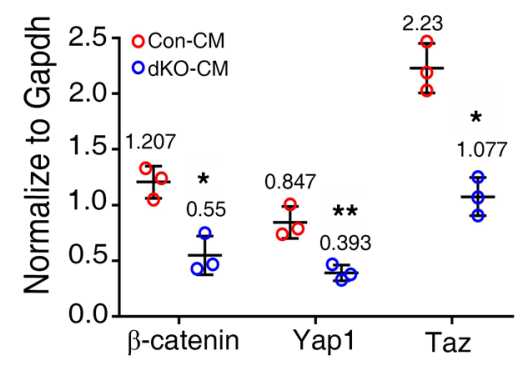

$\mathbf{L}$

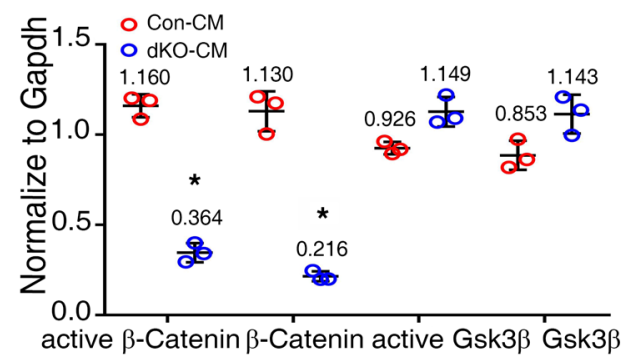

M

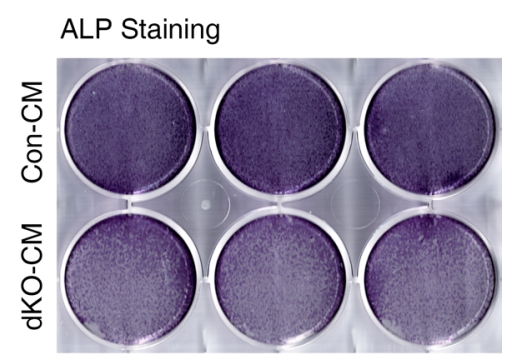

N

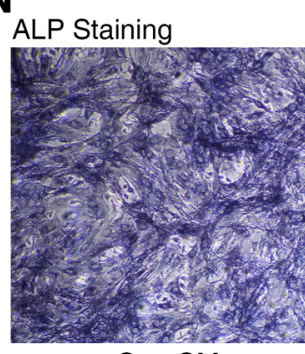

Con-CM

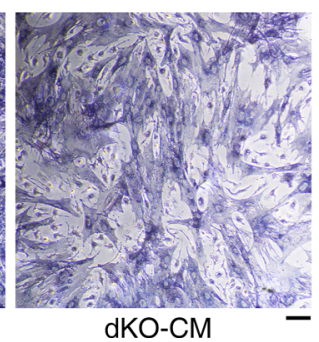

dKO-CM

Figure 5. Pinch loss increases sclerostin expression in osteocytes and indirectly suppresses osteoblast differentiation. (A) Western blot analyses. Protein extracts were isolated from femurs of 6-month-old female control and dKO mice and subjected to Western blotting using an antibody against sclerostin. (B) Quantitative data. $n=3$ mice per group. Unpaired Student's $t$ test. Results are expressed as mean \pm SD. (C) IHC staining of tibial sections of 6-month-old female control and dKO mice with an antibody against sclerostin. Scale bar: $50 \mu \mathrm{m}$. (D) Serum level of sclerostin. Sera were obtained from 6-month-old female control and dKO mice and subjected to ELISA assay for sclerostin. $n=8$ mice per group. Unpaired Student's $t$ test. Results are 
expressed as mean \pm SD. (E) Western blot analysis. Middiaphyseal femoral shafts (with their BM flushed) of 6-month-old female control and dKO mice were cut into small pieces and cultured in serum-free $\alpha$-MEM supplemented with $1 \%$ penicillin/streptomycin for 48 hours and conditioned media (CM) were collected for Western blotting with an antibody against sclerostin. (F-J) Primary BM stromal cells (BMSCs) were isolated from normal C57BL/6 mice and treated with control and mutant CM from E in osteoblast differentiation media ( $\alpha$-MEM containing $10 \%$ FBS, $1 \%$ penicillin/streptomycin, $50 \mu g / m L$ ascorbic acid, and $5 \mathrm{mM} \beta$-glycerophosphate) for 7 days, followed by qPCR analyses for expression of indicated mRNAs, which were normalized to Gapdh mRNA (F) or Western blotting using indicated antibodies (G and I). Quantitative data for G (H) and I (J). (K-N) Primary calvarial osteoblasts were isolated from 3-day-old normal C57BL/6 mice and treated with control and mutant CM in osteoblast differentiation media for 48 hours, followed by Western blotting using indicated antibodies (K) or alkaline phosphatase (ALP) staining ( $\mathbf{M}$ and $\mathbf{N})$. Quantitative data of $\mathbf{K}(\mathbf{L})$. Scale bar: $100 \mu \mathrm{m}$. ${ }^{*} P<0.05,{ }^{*} P<0.01$, ${ }^{* * *} P<0.001$ vs. controls. Unpaired Student's $t$ test. Results are expressed as mean $\pm \mathrm{SD}$. Western blotting analyses in this figure were repeated 3 times.

dKO-CM potently suppressed expression of several important osteoblast genes, including those encoding Runx2, Osx, and Colla1, at both the protein and mRNA levels (Figure 5, F-H). Interestingly, dKO-CM dramatically reduced the protein levels of $\beta$-catenin, Yap1, and Taz in primary BMSC cultures (Figure 5, I and J). Furthermore, dKO-CM dramatically decreased the levels of both total and

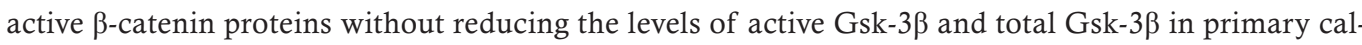
varial osteoblast cultures (Figure 5, $\mathrm{K}$ and $\mathrm{L}$ ). dKO-CM also reduced the alkaline phosphatase (ALP) activity in primary calvarial osteoblast cultures (Figure $5, \mathrm{M}$ and $\mathrm{N}$ ).

Deleting Pinch in osteocytes and mature osteoblasts reduces BMSC proliferation and osteoblast formation. To investigate whether Pinch loss in osteocytes and mature osteoblasts impacts the BM cells, we prepared fresh BM cells of the 2 genotypes and performed colony forming unit-fibroblast (CFU-F) assays and observed reduced number of CFU-F colony in dKO relative to the control group (Figure 6A). Furthermore, Pinch loss also drastically reduced the number of CFU-osteoblast (CFU-OB) assays (Figure 6B). We determined the in vitro proliferation capacity of primary BMSCs from control and $\mathrm{dKO}$ mice by performing the CCK8 assay and observed a reduced proliferation rate in $\mathrm{dKO}$ versus control cells (Figure 6C), which may contribute to the reduced formation of both CFU-F and CFU-OB in $\mathrm{dKO}$ mice.

Pinch deletion increases BM adiposity, and primary BMSCs from dKO mice display decreased osteoblastic, but increased adipogenic, differentiation capacity. Interestingly, we found that primary BMSCs from dKO mice displayed reduced expression of osteoblast differentiation genes, including Runx2, Osterix, Col1 1 1, and ALP activity (Figure 6, D-F). In contrast, dKO BMSCs exhibited increased expression of adipogenic differentiation genes - including those encoding peroxisome proliferator-activated receptor $\gamma$ (Ppar- $\gamma$ ), adipocyte Protein 2 (aP2), and $\mathrm{C} / \mathrm{EBP} \alpha$, but not preadipocyte factor-1 (Pref-1) or adiponectin (Figure 6, G and $\mathrm{H}$ ) and enhanced adipgenic differentiation, as evidenced by Oil Red O staining (Figure 6I). Results from H\&E staining of tibial sections of 6-month-old female control and $\mathrm{dKO}$ mice showed dramatically increased adiposity in dKO BM (Figure 6J). Finally, the expression levels of Yap1 and Taz proteins and mRNAs, which were reported to play a role in controlling the MSC fate (35), were drastically decreased in dKO versus control BMSC cultures (Figure 6, K-M).

Pinch ablation decreases integrin $\beta 3, I L K$, and $\alpha$-parvin; increases active caspase- 3 and - 8 expression; and promotes osteocyte apoptosis. We further performed the TUNEL staining of tibial cross sections of 6-month-old female control and $\mathrm{dKO}$ mice and found that apoptotic osteocytes were scarcely detected in control cortical bones; the number of apoptotic osteocytes was significantly increased in $\mathrm{dKO}$ cortical bones (Figure 7, A-C). We isolated primary osteocytes for flow cytometry analysis to further assess cell apoptosis. To confirm that the isolated cells from cortical bone were $\mathrm{Dmp} 1^{+}$osteocytes, we performed immunofluorescence staining using an anti-Dmp1 antibody. The result showed that almost all cells were Dmp $1^{+}$cells (Figure 7D). Results from flow cytometry analysis of those cells revealed increased apoptosis in $\mathrm{dKO}$ cultures compared with that of control cultures (Figure 7E). Consistently, results from Western blotting revealed that the levels of active caspase- 8 and caspase- 3 proteins, but not their total protein levels, were increased in dKO relative to control osteocytes (Figure 7, F and G). The levels of several Pinch-related proteins, including the ILK, integrin $\beta 3$, and $\alpha$-parvin, were decreased in $\mathrm{dKO}$ bones compared with those of control bones (Figure $7 \mathrm{H}$ ).

Pinch deficiency exacerbates hindlimb unloading-induced bone loss and impairs active ulna loading-induced bone formation. Skeleton throughout life is constantly loaded by mechanical forces generated by daily physical activities and gravity. Osteocytes are believed be the main mechano-sensors of bone. Whereas increased mechanical forces promote bone formation, decreased loading causes bone loss and skeletal fragility. The severe osteopenia of dKO mice prompted us to investigate whether Pinch expression in Dmp-1-expressing cells is involved in mediation of bone mechanotransduction. We first utilized a well-established tail suspension mouse model to create a condition of hindlimb unloading (HLU) as previously described by Robling et al. (39). 
A CFU-F

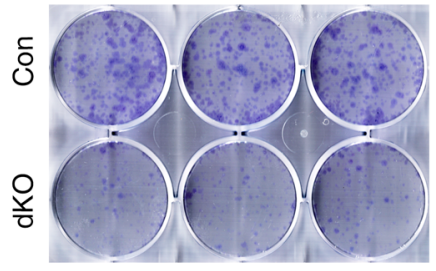

B CFU-OB

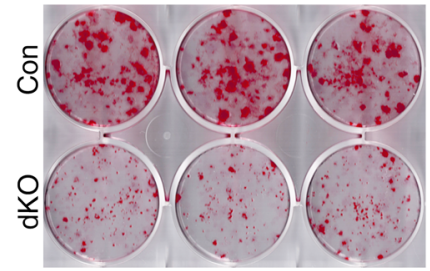

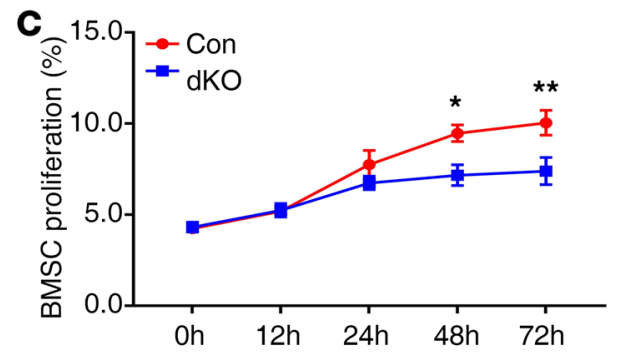

$\mathbf{F}$
D

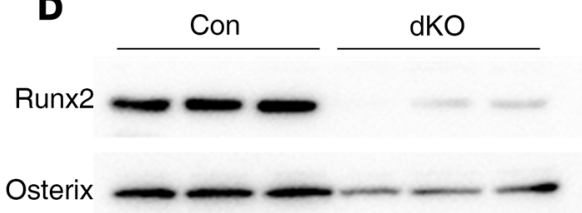

Gapdh
E

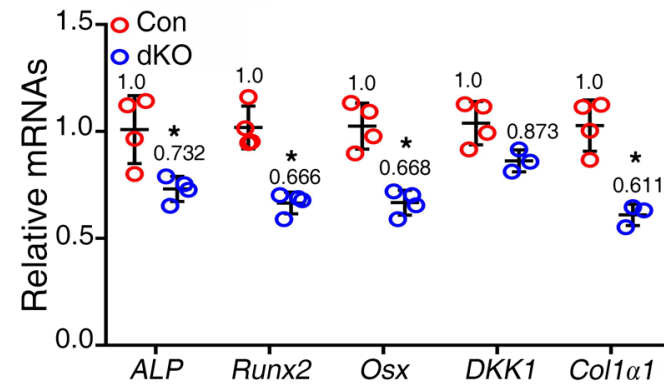

ALP Staining

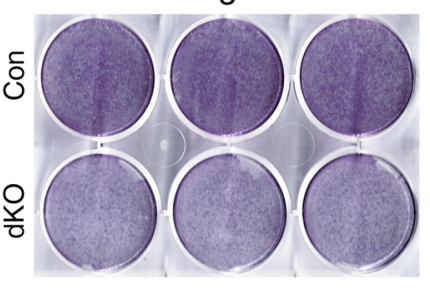

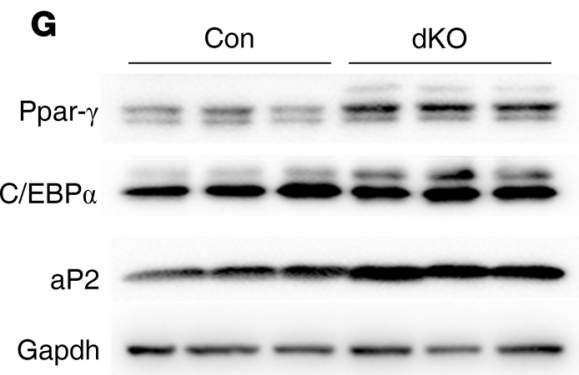

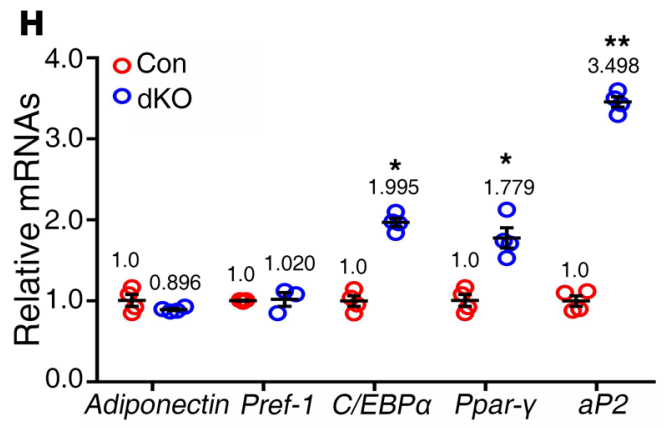

I

Oil Red O Staining

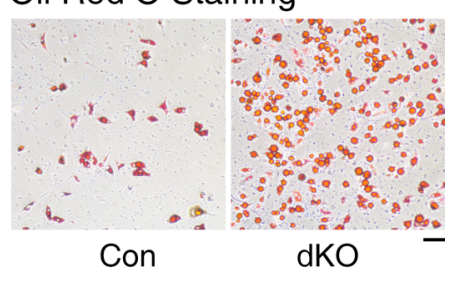

J

H\&E
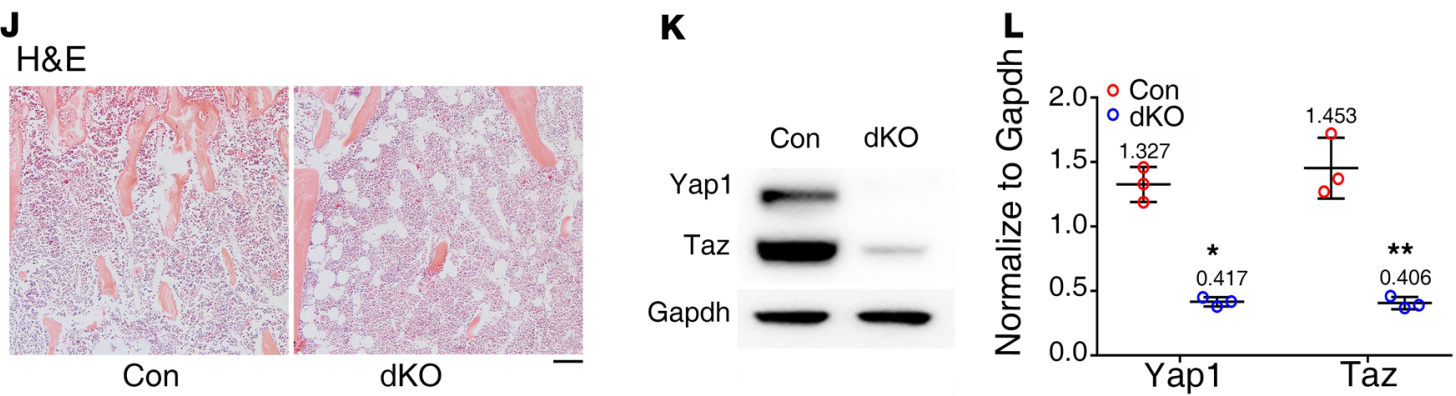
Figure 6. BMSCs from dKO mice display decreased osteoblastic, but increased adipogenic, differentiation capacity. (A) Colony forming unit-fibroblast (CFU-F) assays. $n=6$ mice per group. (B) CFU-osteoblast (CFU-OB) assays. $n=6$ mice per group. (C) BMSC proliferation assay. BMSCs were seeded in a 96-well plate at a density of 2,000 cells/well. The absorbance was measured at the time points of $12,24,48$, and 72 hours. ${ }^{*} P<0.05$, ${ }^{* *} P<0.01$ vs. controls. Unpaired Student's $t$ test. Results are expressed as mean \pm SD. (D-F) In vitro osteoblastic differentiation. Primary BMSCs from 5-month-old female control and dKO mice were seeded in 6-well plates at a density of $4 \times 10^{5}$ cells/well in $2 \mathrm{~mL} \alpha$-MEM media (complete $\alpha$-MEM containing $50 \mu \mathrm{g} / \mathrm{mL}$ L-ascorbic acid) for 7 days. Whole cell extracts were used for Western blot analysis with the indicated antibodies (D), for qPCR analysis $(\mathbf{E})$, or for ALP staining (F). $n=3$ mice for each group. ${ }^{*} P<0.05$ vs. controls. Unpaired Student's $t$ test. Results are expressed as mean \pm SD. (G-I) In vitro adipogenic differentiation. Primary BMSCs from 5-month-old female control and dKO mice were seeded 6-well plates at a density of $4 \times 10^{5}$ cells/well and cultured in adipogenic differentiation media (Stemcell Technology, catalog 05503) for 14 days, followed by Western blot analysis with the indicated antibodies (G), and qPCR analysis (H), or for 21 days, followed by Oil Red 0 staining (I). $n=3$ mice for each group. ${ }^{*} P<0.05$, ${ }^{* *} P<0.01$ vs. controls. Unpaired Student's $t$ test. Results are expressed as mean \pm SD. Scale bar: $100 \mu \mathrm{m}$. (J) BM adiposity. Tibial sections of 6-month-old female control and dKO mice were subjected to H\&E staining. (K and $\mathbf{L}$ ) Western blotting. Protein extracts isolated from BMSCs of 6-month-old female mice of the 2 genotypes were subjected to Western blotting using the indicated antibodies. $n=5$ mice for each group. ${ }^{*} P<0.05$ vs. controls. Unpaired Student's $t$ test. Results are expressed as mean \pm SD. (M) qPCR analysis. Total RNAs were isolated from BMSCs of 6-month-old female mice of the 2 genotypes and subjected to qPCR analysis. $n=3$ mice for each group. ${ }^{*} P<0.05,{ }^{* *} P<0.01$ vs. controls. Unpaired Student's $t$ test. Results are expressed as mean \pm SD. Western blotting analyses in this figure were repeated at least 3 times.

Three-month-old female control and dKO mice were subjected to HLU for 21 days, followed by $\mu \mathrm{CT}$ analysis of distal femurs. The results showed that HLU induced significant bone loss in both control and dKO mice, as demonstrated by significant decreases in BV/TV, Tb.N, and Ct.Th and an increase in Tb.Sp induced by HLU in both control and dKO mice (Figure 8, A-F). However, the magnitudes in the HLU-induced decreases in $\mathrm{BV} / \mathrm{TV}$ and Tb.N were increased in $\mathrm{dKO}$ mice compared with those in control mice $(P<0.05$, Con-HLU/Con versus dKO-HLU/dKO) (Figure 8, B and C). These results suggest that Pinch is probably involved in mechanical unloading-induced bone loss. Next, we utilized an active ulna loading model to determine whether Pinch is required for mechanical loading. In this experiment, 3-month-old female control and $\mathrm{dKO}$ mice were subjected to active mechanical loading as previously described (40). $\mu \mathrm{CT}$ analysis of ulnas of control and $\mathrm{dKO}$ mice with and without loading revealed that the $\mathrm{Ct}$.Th was increased by loading in control ulna; this increase was lost in dKO ulna $(P<0.05$, Con-load/Con versus dKO-load/dKO) (Figure 8G). Results from the calcein double-labeling experiments showed that, although both control and $\mathrm{dKO}$ ulnas responded to loading by increasing MAR and BFR, the magnitudes stimulated by loading were decreased by Pinch loss $(P<0.05$, Con-load/Con versus dKO-load/dKO) (Figure 8, H-J).

\section{Discussion}

In this study, we establish a critical role of Pinch proteins, through expression in mature osteoblasts and osteocytes, in regulation of bone mass and mechanotransduction in mice. We also demonstrate a functional redundancy of Pinch1/2 in control of bone mass. We define distinct cellular and molecular mechanisms through which Pinch regulates bone homeostasis. The findings of this study may provide a potential therapeutic target for the prevention and treatment of metabolic bone diseases, such as osteoporosis.

In this study, we establish that Pinch expression in osteocytes and mature osteoblasts plays a critical role in control of osteoblast function and bone formation. This notion is supported by the following lines of experimental evidence: (a) Pinch loss reduces the serum level of P1NP, which is a bona fide bone formation marker; (b) results from our calcein double-labeling experiments provide direct evidence that the osteoblast-mediated bone forming activity is impaired by Pinch loss, as demonstrated by dramatic decreases in MAR and BFR in dKO versus control mice; (c) reduced osteoid production and deposition and mineralization further point to an impairment in osteoblast number and/or function in dKO mice; and (d) Pinch loss reduces expression of Osx, a marker for preosteoblasts and osteoblasts, in bones. It should be noted that Pinch loss does not markedly impact the Rankl/Opg ratio, osteoclast formation, and bone resorption. Thus, impaired osteoblast formation and function primarily contributes to the osteopenia in mutant mice.

We provide convincing evidence that Pinch loss greatly increases expression of sclerostin in osteocytes, which contributes, at least in part, to the impaired osteoblast formation and function in mutant mice. Indeed, Pinch loss largely increases sclerostin expression in osteocytes embedded in the bone matrix. Of particular interest, $\mathrm{CM}$ from ex vivo cultured mutant bones contain abundant sclerostin and decrease the expression of $\beta$-catenin, Runx2, Osx, Col1a1, and Alp in primary BMSCs and calvarial osteoblasts. It is interesting to note that the level of sclerostin in circulation is not increased in mutant mice; this suggests that Pinch regulates the level of sclerostin primarily in the local bone microenvironment and controls osteoblast function and bone formation. Given the important role of sclerostin in control of bone mass and the fact that osteocytes are a main source of sclerostin $(8,41)$, results of the present study demonstrating that 
A

Tunnel stain

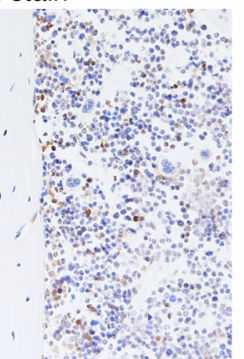

Con

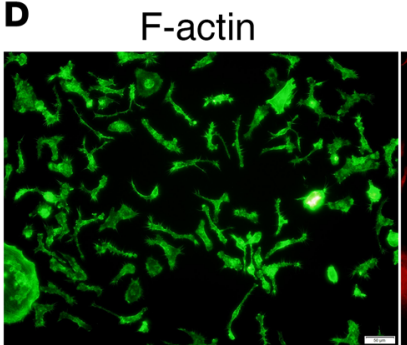

F-actin

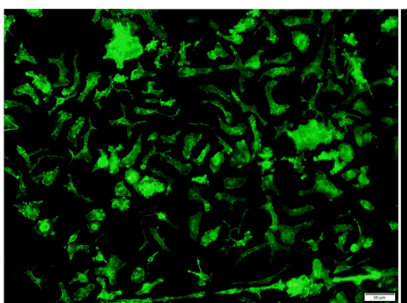

E
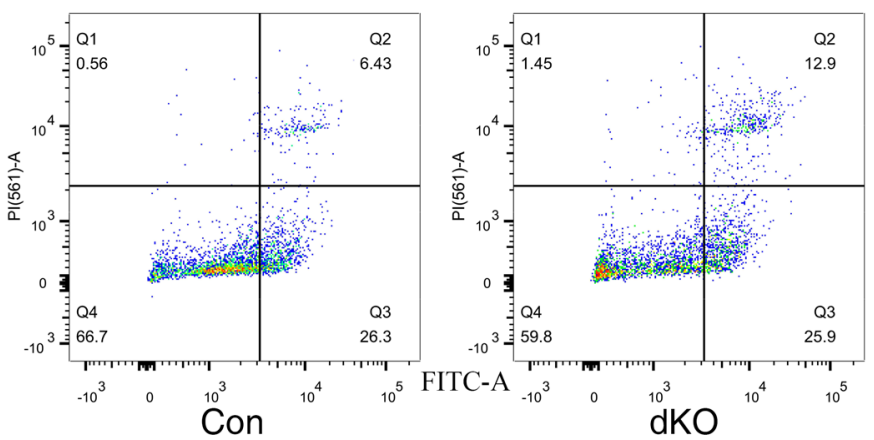

G

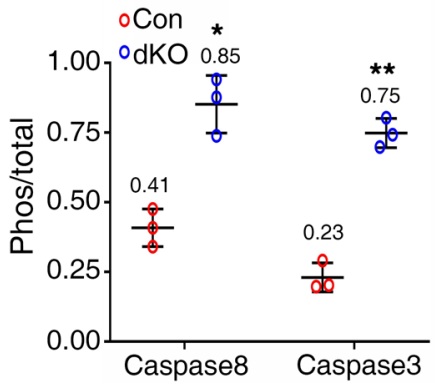

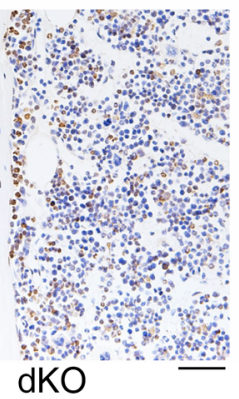

Dmp1 Ab

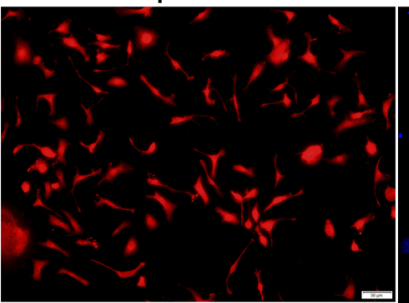

Rabbit IgG
H

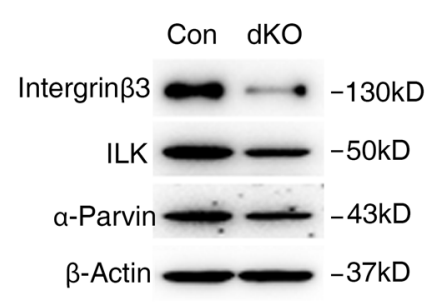

B

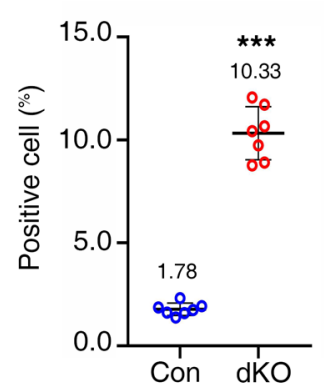

C

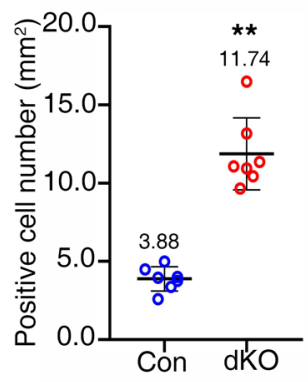

DAPI

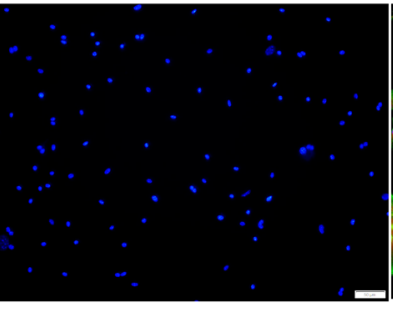

DAPI

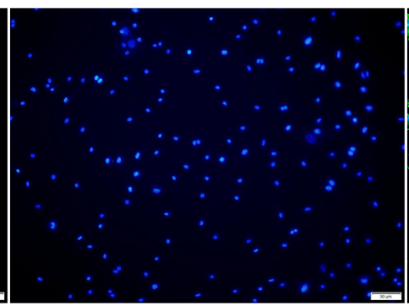

$\mathbf{F}$

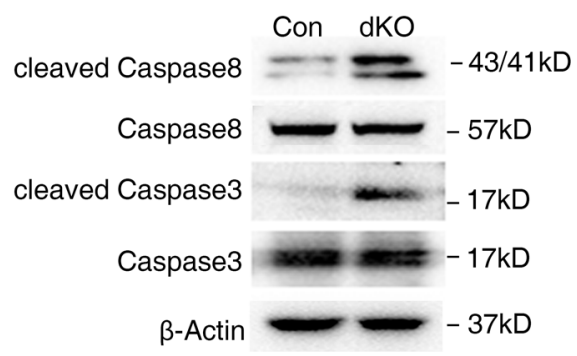

Merged

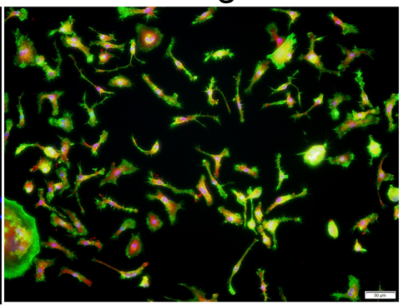

Merged

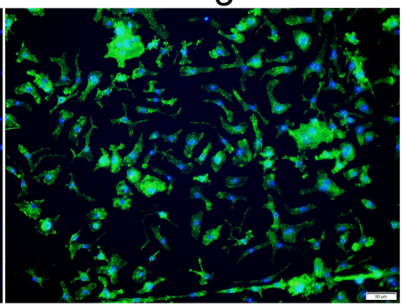

$\beta$-Actin

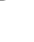


Figure 7. Reduced integrin $\beta 3$, ILK, and $\alpha$-parvin; increased active caspase-3 and -8 expression; and apoptosis in Pinch-deficient osteocytes. (A-C) TUNEL staining. Tibial sections of 6-month-old female control and dKO mice were subjected to TUNEL staining (A), percent apoptotic osteocytes (B), and the number of osteocytes (C) in the cortical bone matrix were quantified. $n=7$ mice per group. ${ }^{* *} P<0.01,{ }^{* *} P<0.001$ vs. controls. Unpaired Student's $t$ test. Results are expressed as mean \pm SD. (D) Primary osteocytes were isolated from middiaphyseal femoral shafts (with their BM flushed) of 9-month-old female control and dKO mice and subjected to immunofluorescence (IF) staining with rabbit DMP1 antibody, normal IgG, or Alexa Fluor 488 Phalloidin (for F-actin) (D). (E) Primary osteocytes from $\mathbf{D}$ were subjected to flow cytometry analysis of cell apoptosis. Scale bar: $20 \mu \mathrm{m}$. (F and $\mathbf{H})$ Western blot analysis. Protein extracts were isolated from middiaphyseal femoral shafts (with their BM flushed) of 6-month-old female control, and dKO mice were subjected to Western blotting analyses using the indicated antibodies. (G) Quantitative data of $\mathbf{F}$. $n=3$. ${ }^{*} P<0.05$, ${ }^{* *} P<0.01$, versus controls, unpaired Student's $t$ test. Results are expressed as mean \pm SD. Western blotting analyses in this figure were repeated at least 3 times.

Pinch proteins function as a critical inhibitory signal for sclerostin expression in osteocytes represents an important finding in osteocyte biology.

Pinch loss in mature osteoblasts and osteocytes indirectly impacts the formation of OFU-F and OFU-OB, cell proliferation, osteoblast gene expression, and differentiation in primary BMSC cultures. These results suggest that the BMSCs are influenced before in vitro experiments in the bone microenvironment by signals initiated by Pinch loss in osteocytes and mature osteoblasts. In addition to reduced osteogenesis, BM and BMSCs of mutant mice display increased adipogenesis. Since there is a discrepancy in literature regarding the role of Yap1/Taz in regulation of MSC differentiation $(35,42)$, whether or not the reduced Yap1/Taz expression in $\mathrm{dKO}$ BMSCs plays a role for the altered BMSC differentiation capacity of those cells remains to be determined.

We utilize 2 unique mouse models to demonstrate an involvement of Pinch in mediation of bone mechanotransduction. We show that Pinch loss exacerbates the HLU-induced bone loss. We further use an active ulna loading mouse model and demonstrate a requirement of Pinch in active ulna loading-induced bone formation in vivo. Batra and coworkers showed that (a) mechanical stress-activated integrin $\alpha 5 \beta 1$ induces opening of connexin 43 (Cx43) hemichannels (43); (b) AKT activation by fluid flow shear stress directly phosphorylates $\mathrm{Cx} 43$ and integrin $\alpha 5$; and (c) Ser-373 of $\mathrm{Cx} 43$ plays a predominant role in mediating the interaction between these 2 proteins and $\mathrm{Cx} 43$ hemichannel opening, a crucial step to mediate the anabolic function of mechanical loading in bone (44). A more recent study showed that IGF1 assembles the formation of a complex between IGF1R and the integrin $\beta 3$ subunit to mediate mechanical stimuli in osteoblastic cells (45). Our results of the present study reveal that Pinch loss reduces expression of integrin $\beta 3$ and 2 integrin-related proteins, ILK and $\alpha$-parvin, in osteocytes. Thus, it is likely that Pinch mediates bone mechanotransduction by modulating, in part, the integrin signaling in osteocytes. Future study will explore this possibility.

Our results suggest that Pinch proteins protect osteocytes from apoptosis and Pinch loss promotes osteocyte apoptosis associated with increased expression of cleaved caspase- 3 and -8 .

Liang and coworkers demonstrate that ablation of either Pinch1 or Pinch 2 in cardiomyocytes in mice does not cause obvious defects in the heart structure and function at the basal level. However, loss of either of the 2 proteins deteriorates myocardial infarction induced by coronary artery ligation. Deletion of both Pinch1/2 in the heart in mice result in severe dilated cardiomyopathy and death due to heart failure (36). In the present study, we demonstrate a functional redundancy of Pinch1/2 in control of bone mass.

In summary, we establish critical roles for Pinch $1 / 2$ in regulation of osteocyte survival and function, bone homeostasis, and bone mechanotransduction, and findings of the present study emphasize the importance of investigating the role of Pinch proteins in human metabolic bone diseases.

\section{Methods}

Animal studies. Dmp1-Cre mice, in which the 10-kb mouse dentin matrix protein 1 (Dmp1) gene promoter drives Cre expression in mature osteoblasts and osteocytes, were previously described (46). Pinch $1^{f l / f l}$ and Pinch $2^{-/-}$mice were previously described (36). To obtain the double mutant mice, Pinch $1^{f / f l}$ mice were first

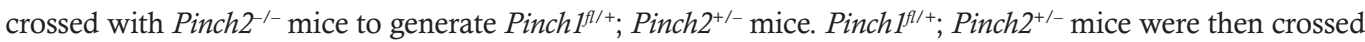
to each other and generate the Pinch $1^{f l f l}$ Pinch $^{-1-}$ mice. Hemizygous Dmp1-Cre transgenic mice were crossed

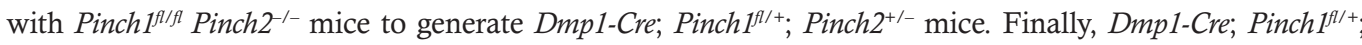

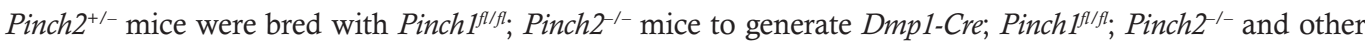
genotypes. All mice used in this study have been crossed with normal C57BL/6 mice for at least 10 generations. All animal experiments were conducted in the specific pathogen free (SPF) Experimental Animal Center of SUSTech. Animals were housed $4 /$ cage at $20^{\circ} \mathrm{C}-24^{\circ} \mathrm{C}$, exposed to a 12 -hour/12-hour light-dark period, and given water and rodent chow ad libitum. Mice were monitored and weighed daily. $\mu \mathrm{CT}$ analysis, 

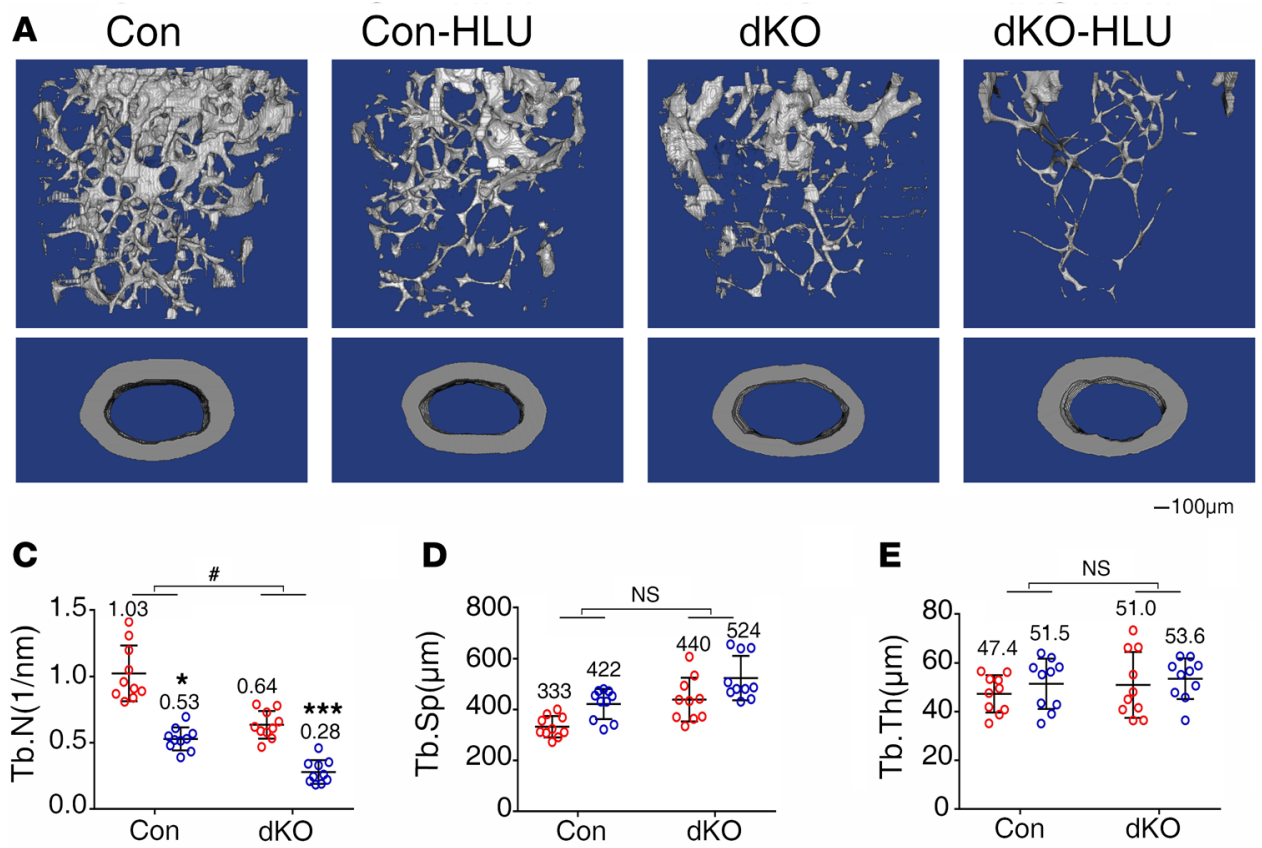

\section{E}
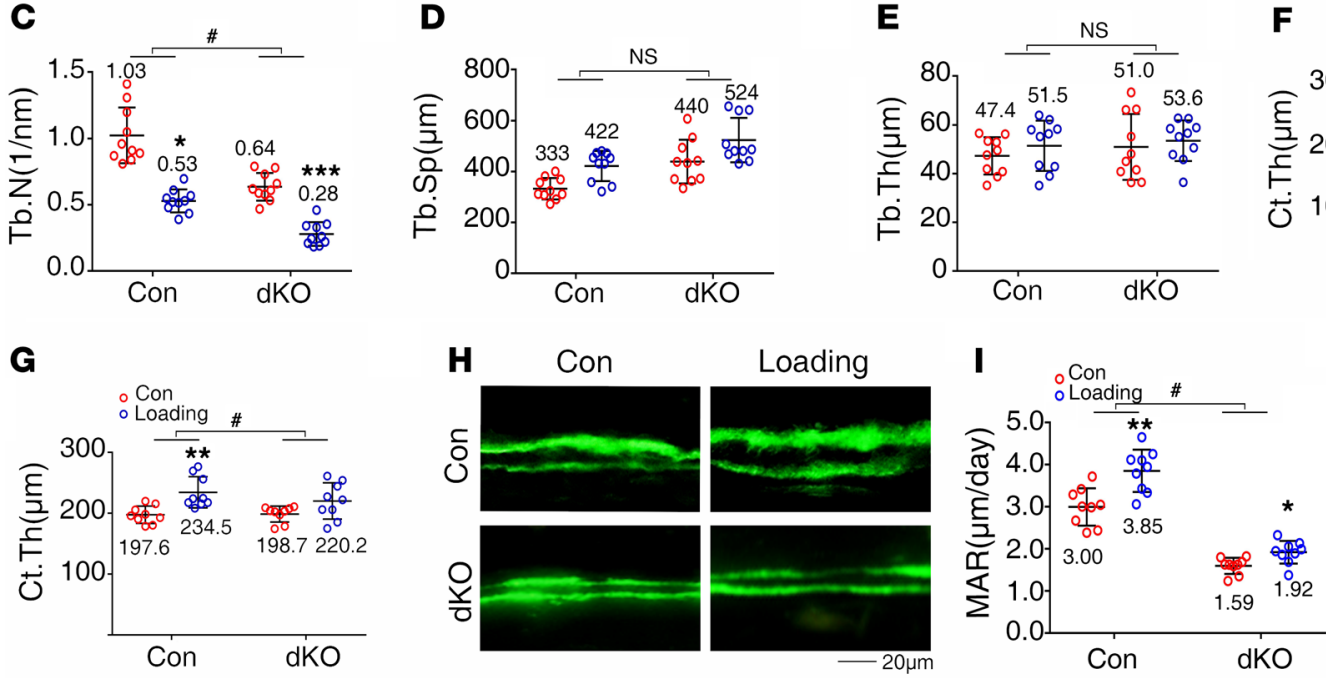
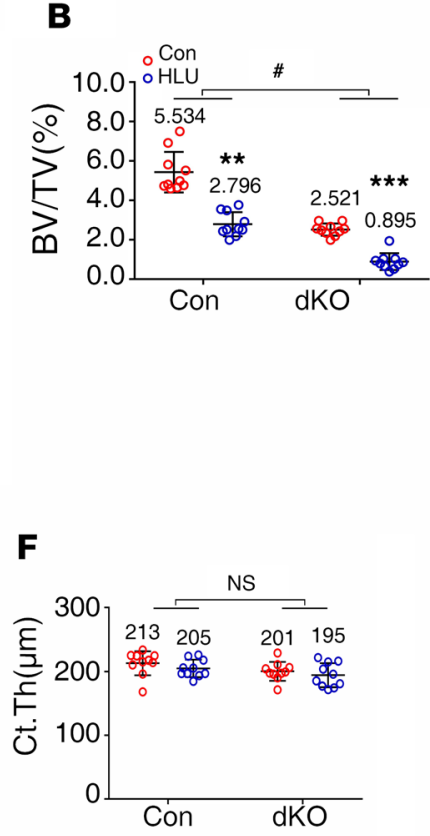

Figure 8. Pinch deletion exacerbates hindlimb unloading-induced bone loss and reduces active ulna loading-stimulated bone formation. (A-F) Hindlimb unloading (HLU) experiment. Three-month-old female control and dKO mice were treated with or without tail suspension for 21 days, followed by $\mu \mathrm{CT}$ analysis of femurs. (A) Three-dimensional (3-D) reconstruction. Scale bar: $100 \mu \mathrm{m}$. (B-F) Quantitative analyses of BV/TV, Tb.N, Tb.Sp, Tb.Th, and Ct.Th. $n=10$ mice per group. ${ }^{*} P<0.05$, ${ }^{* *} P<0.01$, ${ }^{* *} P<0.01$, control (Con) vs. HLU; ${ }^{*} P<0.05$, Con-HLU/Con vs. dKO-HLU/dKO, 2-way ANOVA. Results are expressed as mean \pm SD. (G-J) Ulna loading experiment. Three-month-old female control and dKO mice were subjected to ulna loading as described in Methods. Quantitative analyses for Ct.Th (G). Sections of nondemineralized femurs of control and dKO mice with and without loading were used for measurements of mineralization apposition rate (MAR) and bone formation rate (BFR) (H-J). Quantitative MAR and BFR data for diaphyseal cortical bones. $\mathrm{n}=9$ mice per group. ${ }^{*} P<0.05,{ }^{* *} P<0.01,{ }^{* *} P<0.01$, Con vs. load; ${ }^{*} P<0.05$, Con-load/Con vs. dKO-load/dKO, 2-way ANOVA. Results are expressed as mean \pm SD. Scale bar: $20 \mu \mathrm{m}$.

HLU, and ulna loading are described in detail below. Mouse age and sex and numbers of mice used for each experiment are specified in the figure legends

HLU experiment. Three-month-old female control and $\mathrm{dKO}$ mice were treated with or without tail suspension for 21 days as previously described (39). Mice were euthanized at day 21 by $\mathrm{CO}_{2}$ asphyxiation. Femurs from each group were subjected to $\mu \mathrm{CT}$ analysis as described below.

Ulna loading model. In vivo ulna mechanical loading was performed under anesthesia using a Biomaterial mechanical system Electroforce 3230 (TA Instrument) as described (40). Three-month-old female control and dKO mice commenced unilateral cyclic axial compression of the left ulna at day 1, day 4, day 7, day 10, and day 13. Briefly, 900 cycles were applied at a rate of $4 \mathrm{~Hz}$ with peaking $2.3 \mathrm{~N}$ for 3.75 minutes. Right ulnas were used as contralateral nonloaded controls. Calcein was injected at day 3 and day 10. Mice were euthanized at day 14 by $\mathrm{CO}_{2}$ asphyxiation.

Three-point bending tests. Femurs isolated from 4-month-old control and $\mathrm{dKO}$ female mice were placed in a fixture attached to a materials testing machine and loaded until broken as described (47). This assay primarily tests the mechanical properties of the middiaphysis, which is typically all cortical bone.

Histological evaluation and bone histomorphometry. For histology, bone tissues were fixed in $10 \%$ formalin at $4^{\circ} \mathrm{C}$ for 24 hours, decalcified in $10 \%$ EDTA (pH 7.4) for 10-14 days, and embedded in paraffin. 
Bone sections were used for $\mathrm{H} \& \mathrm{E}$ staining using our standard protocol. Fixed nondemineralized bones were used for $\mu \mathrm{CT}$ analysis in the Department of Biology of SUSTech using a Bruker CT (SkyScan 1172 Micro-CT, Bruker MicroCT) following the standards of techniques and terminology recommended by the American Society for Bone and Mineral Research (ASBMR) (48). For trabecular bone parameters, transverse slices were obtained in the region of interest in the axial direction from the trabecular bone $0.5 \mathrm{~mm}$ below the growth plate (bottom of the primary spongiosa). Contours were defined and drawn close to the cortical bone. The trabecular bone was then removed and analyzed separately. A 3-dimensional analysis was performed on 200 trabecular bone slices. A $0.5-\mathrm{mm}$ section was used to obtain midfemoral cortical bone thickness. The analysis of the specimens involved the following bone measurements: $\mathrm{BMD}, \mathrm{BV} / \mathrm{TV}$, Tb.N, Tb.Th, Tb.Sp, and Ct.Th.

Calcein double labeling, MAR, and BFR. Calcein double labeling, MAR, and BFR were measured as we previously described (49). Briefly, mice were injected i.p. with calcein $(20 \mathrm{mg} / \mathrm{kg})$ at days 6 and 2 before sacrifice. Nondemineralized tibiae were embedded using an Osteo-Bed Bone Embedding kit (EM0200; MilliporeSigma) and sectioned at $5 \mu \mathrm{m}$. Calcein labeling was visualized using EVOS fl microscopy (Invitrogen). Metaphyseal trabecular bones protruding into the BM area and diaphyseal cortical bones were evaluated, and the distance between the double-labeled bands was measured using Image Pro Plus 7.0 software. The MAR was calculated as the mean distance between the double labels divided by the number of the days between the calcein injections. The mineralizing surface per bone surface (MS/BS) was calculated as the percentage of bone surface exhibiting mineralizing activity. MS/BS $=(\mathrm{dLS}+\mathrm{sLS} / 2) / \mathrm{BS}$, where $\mathrm{dLS}$ is the number of double-labeled surfaces and sLS is the number of single-labeled surfaces. The BFR per bone surface (BFR/BS or BFR) is the volume of mineralized bone formed per unit time and per unit bone surface. $\mathrm{BFR} / \mathrm{BS}$ is calculated as $\mathrm{BFR} / \mathrm{BS}=\mathrm{MAR} \times(\mathrm{MS} / \mathrm{BS})$.

IHC and immunofluorescence (IF). IHC staining was performed as we previously described (34). Briefly, 5 - $\mu$ m sections were stained with antibodies or control IgG using the EnVision+System-HRP (DAB) kit (Dako North America Inc.) according to the manufacturer's instruction. For IF, cells were seeded and cultured on sterile glass coverslips in 6-well plates. Twenty-four hours later, cells were fixed in 10\% formaldehyde for 30 minutes at $37^{\circ} \mathrm{C}$. Cells were permeabilized with $0.2 \%$ Triton X-100 containing DAPI for 5 minutes and blocked with 2\% BSA (MilliporeSigma, A1933) for 1 hour. The cells were then stained with a primary antibody overnight at $4^{\circ} \mathrm{C}$. After washing, cells were incubated with anti-mouse Alexa Fluor 594 (Invitrogen) secondary antibodies (1:300) for 1 hour at room temperature. Cells were then imaged using a confocal microscope (SP2-AOBS Leica Microsystems).

TUNEL staining. Cell survival was evaluated using the ApopTag Peroxidase In Situ Apoptosis Detection Kit according to the manufacturer's instruction (EMD Millipore, S7100) as we previously described (34).

Primary BMSC culture and CFU-F and CFU-OB assays. Primary BMSC were isolated from tibiae and femurs of 6-month-old female mice as previously described (49). CFU-F and CFU-OB assays were performed as we previously described (50). Briefly, for the CFU-F assays, BM nucleated cells from 5-monthold female control and $\mathrm{dKO}$ mice were seeded in a 6-well plate with a cell density of $2 \times 10^{6}$ per well and cultured using the Mouse MesenCult proliferation kit (CFU-F assay) for 14 days, followed by Giemsa staining. For the CFU-OB assays, BM nucleated cells from 5-month-old female control and dKO mice were seeded in a 6-well plate at a density of $4 \times 10^{6}$ per well and cultured in osteoblast differentiation media (complete $\alpha$-MEM containing $50 \mu \mathrm{g} / \mathrm{mL}$ L-ascorbic acid and $2.0 \mathrm{mM} \beta$-glycerophosphate) for 21 days (media were changed every 48 hours), followed by alizarin red staining.

Primary calvarial osteoblasts and osteocyte cultures. Primary calvarial osteoblasts were isolated from 3-day-old WT C57 mice. Briefly, calvariae were isolated, and soft tissues were removed as much as possible. Calvariae were then rinsed with cold $1 \times$ PBS 3 times, cut into small pieces, and digested with trypsin for $37^{\circ} \mathrm{C}$ for 30 minutes. The calvariae were rinsed and further digested with $1 \mathrm{mg} / \mathrm{mL}$ of collagenase IA for 60 minutes. Cells were harvested by centrifugation, resuspended, and filtered through a Mesh Of 70 Mesh Filters (Corning, 431751). Cells were cultured in low-glucose DMEM with 10\% FBS (Thermo Fisher Scientific, 16000044) and 1\% penicillin/streptomycin (HyClone, SV30010). For isolation of primary osteocytes from cortical bones, we utilized a protocol that was provided by Lynda Bonewald of the Indiana University School of Medicine (Indianapolis, Indiana, USA). Briefly, middiaphyseal femurs and tibiae of 9-month-old control and $\mathrm{dKO}$ mice were cut into small pieces and digested with collagenase IA at $37^{\circ} \mathrm{C}$ for 30 minutes 3 times and then alternately digested with $5 \mathrm{mM}$ EDTA and collagenase IA at $37^{\circ} \mathrm{C}$ for 30 minutes 6 times. Cells from the last digestion were collected 
for experiments. Cells were then cultured in $\alpha$-MEM (HyClone, SH30265.01) supplemented with $10 \%$ FBS (Thermo Fisher Scientific, 16000044) and 1\% penicillin/streptomycin (HyClone, SV30010).

Osteoclast formation in bone. The TRAP staining of bone sections was performed as we previously described (51). The Oc.S/BS and osteoclast numbers/bone perimeter (Oc.N/BPm) in both primary and secondary spongiosa of tibiae were measured using Image Pro Plus 7.0 software as we previously described (50).

Preparation of conditional media from ex vivo bone tissue cultures. Tibiae and femurs were isolated from 6-month-old control and $\mathrm{dKO}$ mice, and soft tissues were removed. Both ends were cut, and BM was flushed. The remaining cortical bones were cut into small pieces and were cultured in serum-free $\alpha$-MEM media for 48 hours, and CM were collected.

ELISA assays. Serum levels of P1NP were measured using the RatLaps EIA Kit (Immunodiagnostic Systems Limited, catalog AC-33F1) according to the manufacturer's instruction. Serum levels of CTX-1, degradation products from type I collagen during osteoclastic bone resorption, were measured using the RatLaps EIA Kit (Immunodiagnostic Systems Limited, catalog AC-06F1) as previously described (51).

qPCR and Western blotting analyses. Total RNAs were isolated using the RNeasy Mini Kit (QIAGEN, catalog 74104) according to the manufacturer's instruction. RT was performed using $1 \mu \mathrm{g}$ denatured RNA and $100 \mathrm{pmol}$ of random hexamers (Applied Biosystems) in a total volume of $25 \mu \mathrm{L}$ containing 12.5 U MultiScribe reverse transcriptase (Applied Biosystems) as previously described (52). qPCR analysis was performed to measure the relative mRNA levels using the SYBR Green kit (Bio-Rad) as previously described (52). Samples were normalized to Gapdh expression. The DNA sequences of primers used for qPCR are summarized in Supplemental Table 1 (supplemental material available online with this article; https://doi.org/10.1172/jci.insight.131692DS1). Western blot analysis was performed as previously described (53). Briefly, protein extracts were fractionated on a $10 \%$ SDSPAGE gel and transferred onto nitrocellulose membranes (Schleicher \& Schuell). The membrane was blocked in 5\% nonfat milk in Tris-buffered saline/Tween 20 buffer; probed with primary antibodies, followed by incubation with secondary antibodies conjugated with horseradish peroxidase; and visualized using a Western Blotting Detection Kit (GE Healthcare, catalog RPN2106). Antibodies used in this study are listed in Supplemental Table 2.

Statistics. The sample size for each experiment was determined based on our previous experience. Animals used in experiments of this study were randomly grouped. IHC, IF, and histology were performed and analyzed in a double-blinded manner. Statistical analyses were completed using the Prism GraphPad. Unpaired 2-tailed Student's $t$ test (2 groups) and 2-way ANOVA (multiple groups) were used. $P<0.05$ was considered statistically significant.

Study approval. All animal studies were approved by the IACUC of SUSTech.

\section{Author contributions}

GX, HC, and YW designed the study. YW, QY, YZ, XL, SL, PZ, LM, and YL conducted the study and collected data. YW, QY, and HC analyzed data. GX, HC, XB, CW, YW, CL, JQF, and DC interpreted data. GX, HC, and YW drafted the manuscript. GX, HC, and YW take the responsibility for the integrity of the data analysis.

\section{Acknowledgments}

The authors thank the Materials Characterization and Preparation Center (MCPC) at SUSTech for help with instrument training and Huamin $\mathrm{Xu}$ for mouse breeding. This work was supported, in part, by the National Natural Science Foundation of China grants (8163066, 81870532, 81972100), the Guangdong Provincial Science and Technology Innovation Council Grant (2017B030301018), and Science and Technology Innovation Commission of Shenzhen Municipal Government grants (JCYJ20180302174117738, JCYJ20180302174246105, KQJSCX20180319114434843, and JSGG20180503182321166).

Address correspondence to: Guozhi Xiao, Room 341, Faculty Research Building 1, Southern University of Science and Technology, 1088 Xueyuan Road, Shenzhen 518055, China. Phone: 0755.8801.8488; Email: xiaogz@sustc.edu.cn. Or to: Huiling Cao, Rm 312, Faculty Research Building 1, Southern University of Science and Technology, 1088 Xueyuan Road, Shenzhen 518055, China. Phone: 0755.8801.8408; Email: caohl@sustech.edu.cn. 
1. Tatsumi S, et al. Targeted ablation of osteocytes induces osteoporosis with defective mechanotransduction. Cell Metab. 2007;5(6):464-475.

2. Bonewald LF. The amazing osteocyte. J Bone Miner Res. 2011;26(2):229-238.

3. Schaffler MB, Cheung WY, Majeska R, Kennedy O. Osteocytes: master orchestrators of bone. Calcif Tissue Int. 2014;94(1):5-24.

4. Bellido T. Osteocyte-driven bone remodeling. Calcif Tissue Int. 2014;94(1):25-34.

5. Prideaux M, Findlay DM, Atkins GJ. Osteocytes: The master cells in bone remodelling. Curr Opin Pharmacol. 2016;28:24-30.

6. Galea GL, Lanyon LE, Price JS. Sclerostin's role in bone's adaptive response to mechanical loading. Bone. 2017;96:38-44.

7. Goldring SR. The osteocyte: key player in regulating bone turnover. RMD Open. 2015;1(Suppl 1):e000049.

8. Winkler DG, et al. Osteocyte control of bone formation via sclerostin, a novel BMP antagonist. EMBO J. 2003;22(23):6267-6276.

9. Xiong J, Onal M, Jilka RL, Weinstein RS, Manolagas SC, O’Brien CA. Matrix-embedded cells control osteoclast formation. Nat Med. 2011;17(10):1235-1241.

10. Nakashima T, et al. Evidence for osteocyte regulation of bone homeostasis through RANKL expression. Nat Med. 2011;17(10):1231-1234

11. Kramer I, et al. Osteocyte Wnt/beta-catenin signaling is required for normal bone homeostasis. Mol Cell Biol. 2010;30(12):3071-3085.

12. Shimada T, et al. Targeted ablation of Fgf23 demonstrates an essential physiological role of FGF23 in phosphate and vitamin D metabolism. J Clin Invest. 2004;113(4):561-568.

13. Duan P, Bonewald LF. The role of the wnt/ $\beta$-catenin signaling pathway in formation and maintenance of bone and teeth. Int $J$ Biochem Cell Biol. 2016;77(Pt A):23-29.

14. Uda Y, Azab E, Sun N, Shi C, Pajevic PD. Osteocyte Mechanobiology. Curr Osteoporos Rep. 2017;15(4):318-325

15. Jilka RL, Noble B, Weinstein RS. Osteocyte apoptosis. Bone. 2013;54(2):264-271.

16. Li S, et al. PINCH1 regulates cell-matrix and cell-cell adhesions, cell polarity and cell survival during the peri-implantation stage. J Cell Sci. 2005;118(Pt 13):2913-2921.

17. Liang X, et al. PINCH1 plays an essential role in early murine embryonic development but is dispensable in ventricular cardiomyocytes. Mol Cell Biol. 2005;25(8):3056-3062.

18. Fukuda T, Chen K, Shi X, Wu C. PINCH-1 is an obligate partner of integrin-linked kinase (ILK) functioning in cell shape modulation, motility, and survival. J Biol Chem. 2003;278(51):51324-51333.

19. Chen K, Tu Y, Zhang Y, Blair HC, Zhang L, Wu C. PINCH-1 regulates the ERK-Bim pathway and contributes to apoptosis resistance in cancer cells. J Biol Chem. 2008;283(5):2508-2517.

20. Xu H, Cao H, Xiao G. Signaling via PINCH: Functions, binding partners and implications in human diseases. Gene. 2016;594(1):10-15.

21. Sakai T, et al. Integrin-linked kinase (ILK) is required for polarizing the epiblast, cell adhesion, and controlling actin accumulation. Genes Dev. 2003;17(7):926-940.

22. Stanchi F, et al. Consequences of loss of PINCH2 expression in mice. J Cell Sci. 2005;118(Pt 24):5899-5910.

23. Tu Y, Li F, Goicoechea S, Wu C. The LIM-only protein PINCH directly interacts with integrin-linked kinase and is recruited to integrin-rich sites in spreading cells. Mol Cell Biol. 1999;19(3):2425-2434.

24. Meder B, et al. PINCH proteins regulate cardiac contractility by modulating integrin-linked kinase-protein kinase B signaling. Mol Cell Biol. 2011;31(16):3424-3435.

25. Wu C. Integrin-linked kinase and PINCH: partners in regulation of cell-extracellular matrix interaction and signal transduction. J Cell Sci. 1999;112(Pt 24):4485-4489.

26. Chiswell BP, Zhang R, Murphy JW, Boggon TJ, Calderwood DA. The structural basis of integrin-linked kinase-PINCH interactions. Proc Natl Acad Sci USA. 2008;105(52):20677-20682.

27. Kogata N, Tribe RM, Fässler R, Way M, Adams RH. Integrin-linked kinase controls vascular wall formation by negatively regulating Rho/ROCK-mediated vascular smooth muscle cell contraction. Genes Dev. 2009;23(19):2278-2283.

28. Montanez E, Wickström SA, Altstätter J, Chu H, Fässler R. Alpha-parvin controls vascular mural cell recruitment to vessel wall by regulating RhoA/ROCK signalling. EMBO J. 2009;28(20):3132-3144.

29. Akhtar N, Streuli CH. An integrin-ILK-microtubule network orients cell polarity and lumen formation in glandular epithelium. Nat Cell Biol. 2013;15(1):17-27.

30. Kovalevich J, Tracy B, Langford D. PINCH: More than just an adaptor protein in cellular response. J Cell Physiol. 2011;226(4):940-947.

31. Wang-Rodriguez J, Dreilinger AD, Alsharabi GM, Rearden A. The signaling adapter protein PINCH is up-regulated in the stroma of common cancers, notably at invasive edges. Cancer. 2002;95(6):1387-1395.

32. Zhang JT, et al. Up-regulation of PINCH in the stroma of oral squamous cell carcinoma predicts nodal metastasis. Oncol Rep. 2005;14(6):1519-1522.

33. Eke I, et al. PINCH1 regulates Akt1 activation and enhances radioresistance by inhibiting PP1alpha. J Clin Invest. 2010;120(7):2516-2527.

34. Wu C, et al. Kindlin-2 controls TGF- $\beta$ signalling and Sox9 expression to regulate chondrogenesis. Nat Commun. $2015 ; 6: 7531$.

35. Guo L, et al. Kindlin-2 regulates mesenchymal stem cell differentiation through control of YAP1/TAZ. J Cell Biol. 2018;217(4):1431-1451.

36. Liang $\mathrm{X}$, et al. Targeted ablation of PINCH1 and $\mathrm{PINCH} 2$ from murine myocardium results in dilated cardiomyopathy and early postnatal lethality. Circulation. 2009;120(7):568-576.

37. Li X, et al. Sclerostin binds to LRP5/6 and antagonizes canonical Wnt signaling. J Biol Chem. 2005;280(20):19883-19887.

38. Semënov M, Tamai K, He X. SOST is a ligand for LRP5/LRP6 and a Wnt signaling inhibitor. J Biol Chem. 2005;280(29):26770-26775.

39. Robling AG, et al. Mechanical stimulation of bone in vivo reduces osteocyte expression of Sost/sclerostin. J Biol Chem. 2008;283(9):5866-5875.

40. Morse A, Schindeler A, McDonald MM, Kneissel M, Kramer I, Little DG. Sclerostin Antibody Augments the Anabolic Bone Formation Response in a Mouse Model of Mechanical Tibial Loading. J Bone Miner Res. 2018;33(3):486-498.

41. Cosman F, et al. Romosozumab Treatment in Postmenopausal Women with Osteoporosis. N Engl J Med. 2016;375(16):1532-1543. 
42. Xiong J, Almeida M, O'Brien CA. The YAP/TAZ transcriptional co-activators have opposing effects at different stages of osteoblast differentiation. Bone. 2018;112:1-9.

43. Batra N, et al. Mechanical stress-activated integrin $\alpha 5 \beta 1$ induces opening of connexin 43 hemichannels. Proc Natl Acad Sci USA. 2012;109(9):3359-3364.

44. Batra N, Riquelme MA, Burra S, Kar R, Gu S, Jiang JX. Direct regulation of osteocytic connexin 43 hemichannels through AKT kinase activated by mechanical stimulation. J Biol Chem. 2014;289(15):10582-10591.

45. Tahimic CG, et al. Regulation of Ligand and Shear Stress-induced Insulin-like Growth Factor 1 (IGF1) Signaling by the Integrin Pathway. J Biol Chem. 2016;291(15):8140-8149.

46. Lu Y, Xie Y, Zhang S, Dusevich V, Bonewald LF, Feng JQ. DMP1-targeted Cre expression in odontoblasts and osteocytes. J Dent Res. 2007;86(4):320-325.

47. Willinghamm MD, Brodt MD, Lee KL, Stephens AL, Ye J, Silva MJ. Age-related changes in bone structure and strength in female and male BALB/c mice. Calcif Tissue Int. 2010;86(6):470-483.

48. Bouxsein ML, Boyd SK, Christiansen BA, Guldberg RE, Jepsen KJ, Müller R. Guidelines for assessment of bone microstructure in rodents using micro-computed tomography. J Bone Miner Res. 2010;25(7):1468-1486.

49. Zhu K, et al. Impaired bone homeostasis in amyotrophic lateral sclerosis mice with muscle atrophy. J Biol Chem. 2015;290(13):8081-8094.

50. Xiao G, et al. Critical role of filamin-binding LIM protein 1 (FBLP-1)/migfilin in regulation of bone remodeling. $J$ Biol Chem. 2012;287(25):21450-21460.

51. Cao H, et al. Activating transcription factor 4 regulates osteoclast differentiation in mice. J Clin Invest. 2010;120(8):2755-2766

52. Zhu K, et al. ATF4 promotes bone angiogenesis by increasing VEGF expression and release in the bone environment. $J$ Bone Miner Res. 2013;28(9):1870-1884.

53. Gao H, et al. Lipoatrophy and metabolic disturbance in mice with adipose-specific deletion of kindlin-2. JCI Insight 2019;4(13):128405. 